\title{
Article \\ Petrogenesis of Neoproterozoic Ultramafic Rocks, Wadi Ibib-Wadi Shani, South Eastern Desert, Egypt: Constraints from Whole Rock and Mineral Chemistry
}

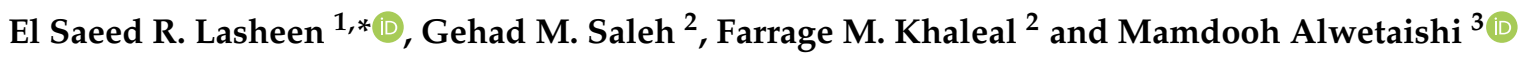 \\ 1 Geology Department, Faculty of Science, Al-Azhar University, Cairo P.O. Box 11884, Egypt \\ 2 Nuclear Materials Authority, El Maadi, Cairo P.O. Box 530, Egypt; drgehad_m@yahoo.com (G.M.S.); \\ farragegeo@yahoo.com (F.M.K.) \\ 3 Department of Civil Engineering, College of Engineering, Taif University, P.O. Box 11099, \\ Taif 21944, Saudi Arabia; m.alwetaishi@tu.edu.sa \\ * Correspondence: elsaeedlasheen@azhar.edu.eg
}

check for

updates

Citation: Lasheen, E.S.R.; Saleh, G.M.; Khaleal, F.M.; Alwetaishi, M. Petrogenesis of Neoproterozoic

Ultramafic Rocks, Wadi Ibib-Wadi Shani, South Eastern Desert, Egypt: Constraints from Whole Rock and Mineral Chemistry. Appl. Sci. 2021, 11, 10524. https://doi.org/10.3390/ app112210524

Academic Editor: Lola Pereira

Received: 24 September 2021

Accepted: 2 November 2021

Published: 9 November 2021

Publisher's Note: MDPI stays neutral with regard to jurisdictional claims in published maps and institutional affiliations.

Copyright: (c) 2021 by the authors. Licensee MDPI, Basel, Switzerland. This article is an open access article distributed under the terms and conditions of the Creative Commons Attribution (CC BY) license (https:/ / creativecommons.org/licenses/by/ $4.0 /)$.

\begin{abstract}
This contribution deals with new geology, petrography, and bulk-rock/mineral chemistry of the poorly studied ultramafics of Wadi Ibib-Wadi Shani (WI-WS) district, South Eastern Desert, Egypt. These ultramafics are dismembered ophiolitic rocks that can be subdivided into serpentinites and serpentinized peridotites. Primary minerals such as olivine and pyroxene are absent in serpentinites, but relics of them occur in serpentinized peridotites. Pseudomorph after olivine is indicated by common hourglass textures with less mesh, whilst schistose bastites reflect a pyroxene pseudomorph. Chromite can be subdivided into Cr-spinel and Al-spinel. Cr-spinel ranges from chromite to magnesochromite in composition, whereas Al-spinel belongs to the spinel field. Cr-spinel includes $\mathrm{YCr}\left(\mathrm{Cr} /\left(\mathrm{Cr}+\mathrm{Al}+\mathrm{Fe}^{+3}\right), \mathrm{YAl}\left(\mathrm{Al} /\left(\mathrm{Al}+\mathrm{Cr}+\mathrm{Fe}^{+3}\right)\right.\right.$, and $\mathrm{YFe}^{+3}\left(\mathrm{Fe}^{+3} /\left(\mathrm{Fe}^{+3}+\mathrm{Al}+\mathrm{Cr}\right)\right.$, similar to forearc peridotite, whilst Al-spinel is more similar to abyssal peridotite, and may be formed during inanition of subduction processes in proto forearc environments. The main secondary minerals are tremolite, talc, and chlorite- - which is subdivided into pycnochlorite and diabantite-and their temperature ranges from 174 to $224^{\circ} \mathrm{C}$. The examined rocks had undergone high partial melting degrees $(>25 \%)$, as indicated by the $\mathrm{Cr} \#$ of their unaltered cores (Cr-spinel, $>0.6)$, whole rocks $\left(\mathrm{Al}_{2} \mathrm{O}_{3}\right.$, $\mathrm{SiO}_{2}, \mathrm{CaO}$, and $\mathrm{MgO}$ ), trace and REEs, depleted $\mathrm{Na}_{2} \mathrm{O}, \mathrm{Al}_{2} \mathrm{O}_{3}$, and $\mathrm{Cr}_{2} \mathrm{O}_{3}$ of clinopyroxene, and high forsterite content $((\mathrm{Fo}=100 \mathrm{Mg} / \mathrm{Mg}+\mathrm{Fe})$, av. $95.23 \mathrm{~mol} \%)$, consistent with forearc settings.
\end{abstract}

Keywords: serpentinization; element mobility; partial melting; forearc affinity

\section{Introduction}

Neoproterozoic ultramafic rocks are commonly distributed in central and southern sectors of the Egyptian Eastern Desert (ED) in the northern Nubian Shield. The Nubian Shield is considered the western side of the Arabian Shield, both of which are juvenile crusts forming the Arabian-Nubian Shield (ANS) of the northern East African Orogeny (EAO) [1]. These rocks constitute 5.3\% of Egypt's crystalline outcrops, mostly as allochthonous nappes [2-6]. The field and geochemical signatures of the ultramafic magma provide clues as to the different tectonic settings. They are either older, dismembered ophiolitic fragments-which are partially or completely metamorphosed to serpentinite, mostly consisting of chromitites and magnesites of mid-ocean ridges or supra-subduction settings-or younger, unmetamorphosed ones [7,8]. The latter, with an age > $640 \mathrm{Ma}$ [9], encompass fresh, concentrically arc-zoned dunites and clinopyroxenites of Alaskan type, mostly consisting of PGEs, Ni-Cu sulfide deposits, and fresh layered intrusions hosting Fe-Ti oxides [10,11].

Ophiolites are slices of the oceanic lithosphere thrust on the continental plates, which help in recognizing the tectonic processes in the mantle [12]. Serpentinites, carbonatized 
serpentinites, talc-carbonates, and listwaenite series (carbonate-rich, silica-carbonate, and birbirites) rocks are the main alteration products of the ophiolitic harzburgite/dunite fragments, due to interaction with $\mathrm{CO}_{2}$ - and $\mathrm{SiO}_{2}$-rich fluids [13]. These processes are widely observed along faults and shear zones. Ultramafic rocks are enriched with magnesium and iron silicate minerals; therefore, carbonation processes take place via the hydrolysis of these minerals, through eviction of $\mathrm{Si}$ and combination of these cations with carbonates $[3,14]$. The tectonic setting of Egyptian ophiolitic mantel rocks (ultramafics) is still a subject of debate; some authors suggest mid-ocean ridge (MOR), while others suggest supra-subduction zone (SSZ) [4,15].

MORs form abyssal mantle rocks of anhydrous melting at low degrees (5-15\%), whilst more than $20 \%$ represents a high degree of melting, forming refractory or depleted rocks in SSZs [16]. The melting degree of the first type depends on the interaction between mantle rocks and melts, as well as the temperature and pressure at melting depth. Conversely, the melting degree of the latter represents a complex process, and depends on the interaction between melts and fluids generated from the subducted slab [17]. In this paper, new geological, mineralogical, and geochemical data are discussed, with a view to detecting the hydrothermal effects, origins and tectonic setting of the studied ultramafic rocks.

\section{Geological Setting}

The Neoproterozoic Era was an important period of crustal growth-especially in the ANS [1], where the Gondwana supercontinent merged. Ophiolites are oceanic lithosphere remnants formed during the Tonian-Cryogenian in mid-ocean ridges (MORs) or through successive subduction and accretion in supra-subduction zone (SSZ) settings during the closing of the Mozambique Ocean [1-8,18]. In Egypt, the ANS Pan-African ophiolites comprise serpentinites from the ED's central and southern sectors (CED and SED, respectively), extending across the Sudanese border (Figure 1a,b). Neoproterozoic ophiolitic rocks of the Wadi Ibib-Wadi Shani (WI-WS) district represent the southwestern extension of the largest Gerf nappe-a dismembered ophiolite sequence covered by metamorphosed, lowered units of ultramafic, metagabbro, and metasedimentary rocks (Figure 1c).

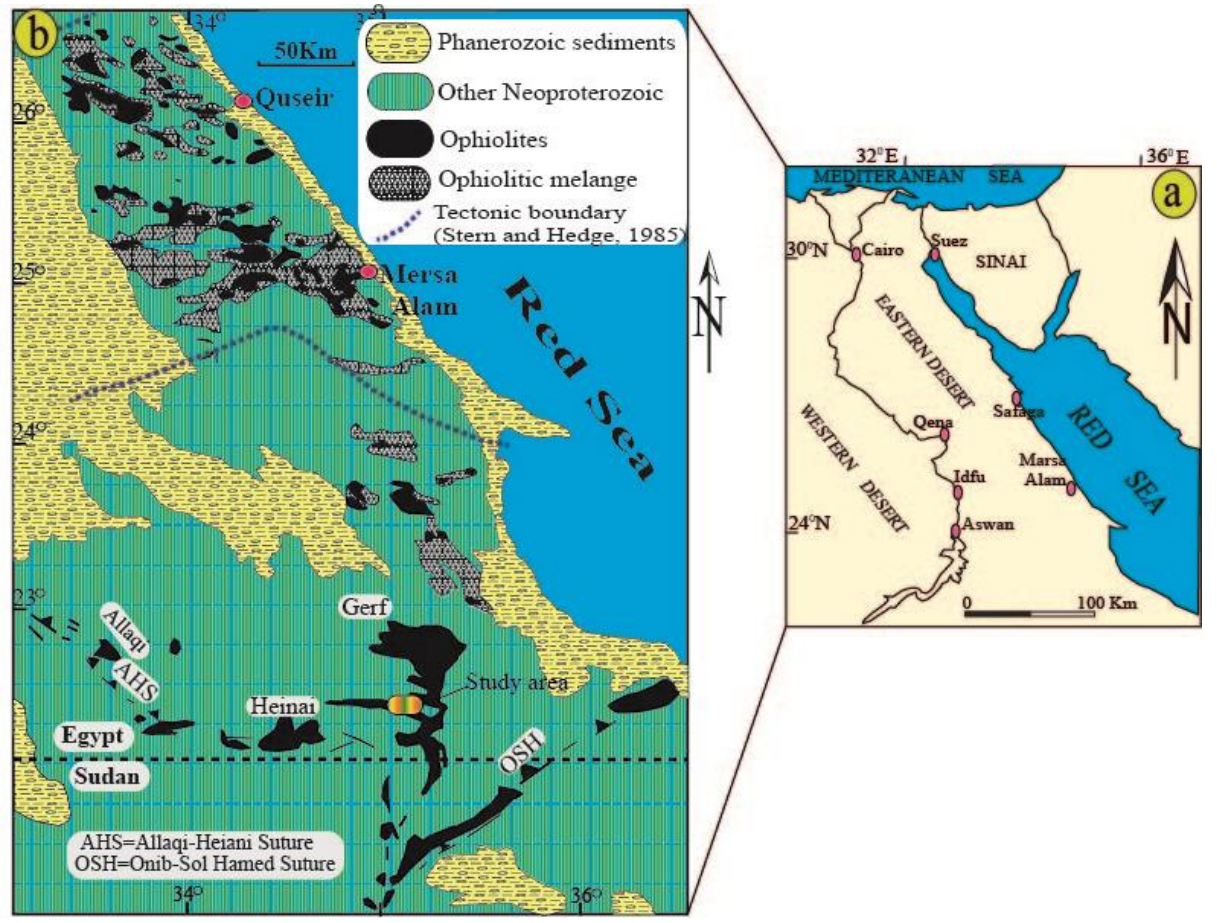

Figure 1. Cont. 


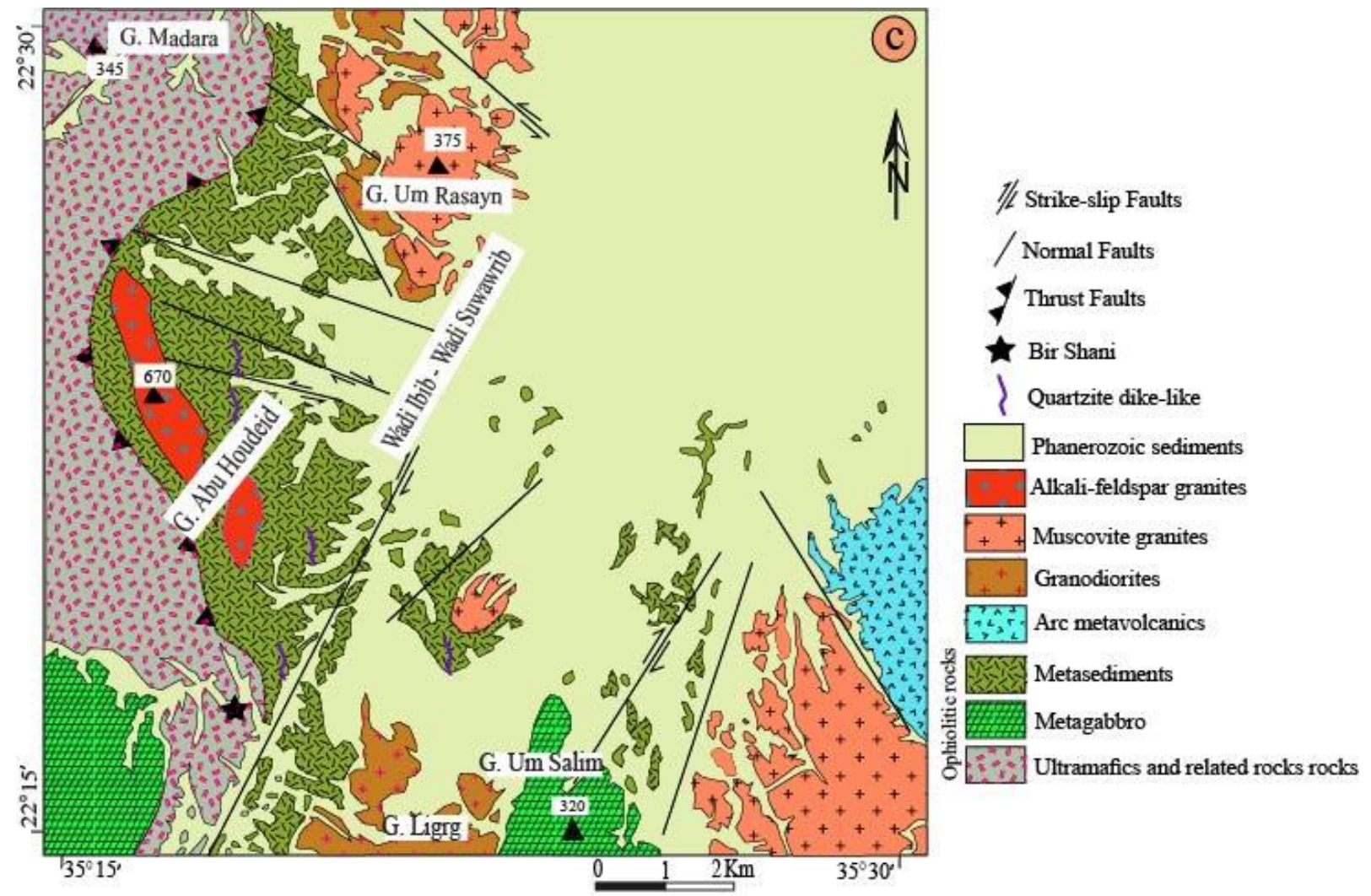

Figure 1. (a) Regional geological map of Egypt. (b) Distribution of Tonian ophiolite rocks [15]. In addition, location of the examined area, and (c) detailed geological map of Wadi Ibib (WI)-Wadi Suwawrib (WS) [19].

The examined ophiolitic ultramafic rocks are represented by the dominant serpentinite and less serpentinized peridotites, which are usually metamorphosed to greenschist facies. Field observations reveal that the examined ultramafic rocks cover $\sim 100 \mathrm{~km}^{2}$, forming an extended N-S ridge with a high relief (950-1100 $\mathrm{m}$ above sea level) at the north-south side of G. Abu Hadeida. They are exposed as mountainous blocks, which are mainly massive and grey-black in color. Furthermore, they exhibit a closely spaced system of joints trending NE-SW and NW-SE along the fault plane. They are thrust over the metasediments (Figure 2a), as indicated by dip directions, with some shearing and tightness of foliation close to the contact. These metasediments are fine-grained, highly foliated, laminated, and exhibit alternating mafic-rich and -poor layers of schists (Figure $2 \mathrm{~b}$ ). Ultramafics are partially to extensively sheared and highly altered to foliated (asbestiform), buff colored, talc-carbonate, carbonate-rich (listwaenite), and magnesite rocks-especially along fault planes and shear zones. These alteration products (listwaenite) occur either as patches or as dike-like with high relief relative to the host (serpentinite)—especially along fault planes. 



Figure 2. (a) Thrust contact between serpentinites and metasediments, and (b) pod of talc-carbonate within serpentinites along the shear zone.

\section{Materials and Methods}

Representative samples were crushed and pulverized using a vibration mill. Loss of ignition (LOI) was measured by the difference in weight after ignition. Whole-rock analysis (major, trace elements, and rare earth elements) of 25 samples was carried out via inductively coupled plasma emission spectrometry (ICP-ES) at Acme Lab, Vancouver, Canada. Detection limits for trace elements and major oxides were $0.01-0.5 \mathrm{ppm}$ and $0.001-0.04 \mathrm{wt} . \%$, respectively. The analytical precision, as calculated from replicate analyses, was $0.5 \%$ for major oxides, and varied from $2 \%$ to $20 \%$ for trace elements. Some samples were selected and analyzed for major elements (as a test) using fused pellets prepared as described in [20], using lithium tetraborate as a flux at the laboratories of the Nuclear Materials Authority (NMA), Cairo, Egypt. The mineral chemistry was carried out at the Microscopy and Microanalyses Facility, University of New Brunswick (UNB), Fredericton, New Brunswick, Canada. Samples were analyzed with a JEOL JXA-733 Electron Microprobe, equipped with ds spec and dQant32 automation (Geller Micro Analytical Labs, Canada). An accelerating voltage of $15 \mathrm{kV}$ and a probe current of $30 \mathrm{nA}$ were used. Peak counting times were $30 \mathrm{~s}$ ( $\mathrm{Al}$ and $\mathrm{Si}), 90 \mathrm{~s}(\mathrm{Ti}, \mathrm{V}, \mathrm{Cr}$, and Cs), and $120 \mathrm{~s}(\mathrm{Na}, \mathrm{Mg}, \mathrm{K}$, $\mathrm{Ca}, \mathrm{Mn}, \mathrm{Fe}$, and $\mathrm{Ni}$ ). High and low backgrounds were counted for one-half of the peak counting times.

\section{Petrography}

Generally, the examined mantle units can be divided into serpentinites and serpentinized peridotites, based on their mineralogical composition. Petrographic examination of 31 samples was carried out at NMA using a polarizing microscope (Olympus bx53). Serpentinites are commonly sheared (carbonatized), and exhibit pseudomorphic textures after olivine and pyroxene minerals. Pseudomorph after olivine is indicated by common hourglass textures with less mesh, whilst bastite reflects pyroxene pseudomorph. Primary minerals such as olivine and pyroxene are completely replaced by antigorite, magnetite, and carbonate minerals. Antigorite occurs as dense sherds of fibro-lamellar, irregular aggregates, ranging from colorless to pale green, fractured, and occupied by iron oxides and carbonates. Abundance of bastites suggest pyroxene pseudomorph, providing evidence for harzburgite protolith (Figure 3a). Scattered patches of carbonate are the main component in the examined serpentinites. Opaque minerals are essentially represented by magnetite (Figure 3b). Magnetite is disseminated as fine, black, anhedral crystals. 

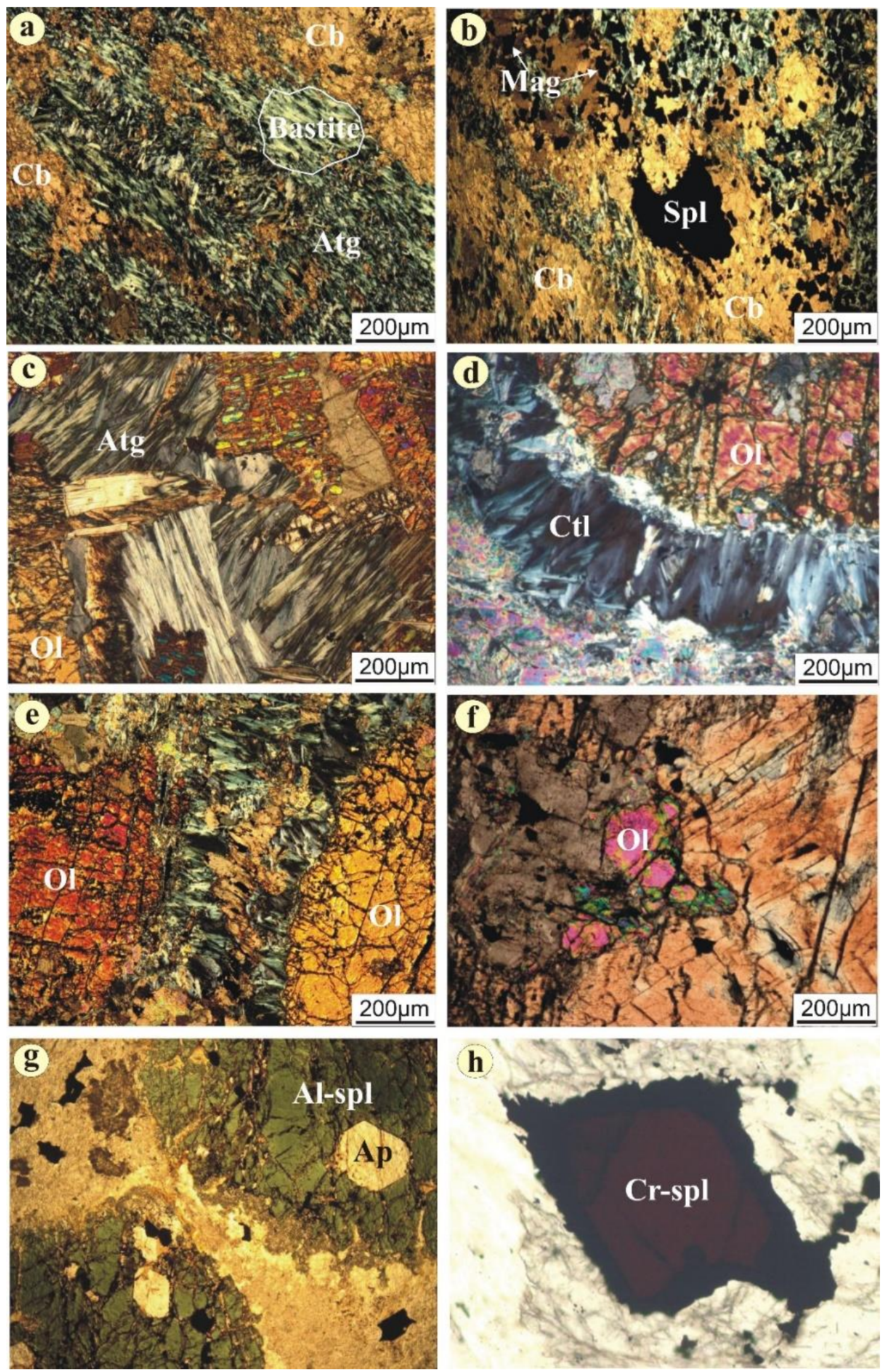

Figure 3. Photomicrographs of serpentinites-(a) sherds of antigorite (Atg) forming a bastite texture after pyroxene; (b) patches of carbonates $(\mathrm{Cb})$ associated with disseminated magnetite (Mag) and serpentinized peridotites: (c) fibro-lamellar antigorite enclosed between olivine (Ol) crystals; (d) perfect veinlet of chrysotile $(\mathrm{Ctl})$; (e) replacement of olivine by antigorite; (f) schiller structure of orthopyroxene (Opx); (g) two porphyroblasts of green spinel (Al-Spl) engulfing apatite (Ap); and (h) fresh core of $\mathrm{Cr}$-spinel $(\mathrm{Cr}-\mathrm{Spl})$ rimmed by secondary $\mathrm{Cr}$-magnetite (Cr-Mag).

On the other hand, serpentinized peridotites are essentially composed of serpentines (antigorite and chrysotile) with variable relics of orthopyroxene, clinopyroxene, olivine, 
and spinels. Tremolite, carbonates, and talc are the main secondary minerals. Fibrolamellar, irregular aggregate of antigorites form asbestiform and hourglass, reflecting their olivine pseudomorph (Figure $3 \mathrm{c}$ ). Chrysotile commonly occurs as veinlets crosscutting olivine crystals (Figure 3d). Olivines reveal significant cracks, and are replaced partially to completely by serpentine, magnetite, and talc along their peripheries, forming iddingsite (Figure 3c-e). Orthopyroxene is replaced by serpentine, chlorite, tremolite, and magnetite, forming schiller structures (Figure 3f). Magnetite and red and green spinels are the main opaque minerals (Figure 3g). Red spinels occur as euhedral, unfractured grains, and are commonly zoned with fresh $\mathrm{Cr}$-spinel cores (red) rimmed by $\mathrm{Cr}$-magnetite (black in color, Figure $3 \mathrm{~h})$.

\section{Results}

\subsection{Mineral Chemistry}

Electron microprobe analysis (EMPA) results of the examined olivine are listed in Table 1. They contain forsterite $(\mathrm{Fo}=100 \mathrm{Mg} / \mathrm{Mg}+\mathrm{Fe}$ ) ranges from 91.94 to 98.13 , with a mean value of $95.23 \mathrm{~mol} \%$. The Fo content of the examined olivine is higher than those reported in [21] (88-92 mol\%), and similar to those of Egyptian ophiolitic peridotites (89-96 mol\%) [22]. Moreover, they possess $\mathrm{NiO}$ and $\mathrm{MnO}$ contents $(0.28-0.92$; $0.087-0.92 \mathrm{wt} . \%$ ) similar to olivine mantle array (plot in and close to mantle array field; Figure $4 a)$.

Table 1. Representative microprobe analysis of olivine.

\begin{tabular}{|c|c|c|c|c|c|c|c|c|c|c|c|c|c|c|c|c|c|c|c|c|}
\hline \multicolumn{21}{|c|}{ Serpentinized Peridotites } \\
\hline No. & 1 & 2 & 3 & 4 & 5 & 6 & 7 & 8 & 9 & 10 & 11 & 12 & 13 & 14 & 15 & 16 & 17 & 18 & 19 & 20 \\
\hline $\mathrm{SiO}_{2}$ & 41.79 & 41.98 & 40.97 & 39.12 & 40.12 & 42.15 & 40.66 & 41.31 & 42.33 & 40.66 & 40.29 & 40.36 & 41.69 & 41.36 & 40.59 & 42.32 & 42.77 & 41.36 & 40.62 & 41.68 \\
\hline $\mathrm{TiO}_{2}$ & 0.01 & 0.02 & 0.01 & 0.03 & 0.01 & 0.02 & 0.01 & 0.02 & 0.01 & 0.01 & 0.01 & 0.02 & 0.01 & 0.02 & 0.02 & 0.02 & 0.03 & 0.02 & 0.01 & 0.02 \\
\hline $\mathrm{Al}_{2} \mathrm{O}_{3}$ & 0.00 & 0.00 & 0.04 & 0.05 & 0.02 & 0.01 & 0.00 & 0.00 & 0.01 & 0.03 & 0.26 & 0.46 & 0.45 & 0.72 & 0.52 & 0.22 & 0.18 & 0.15 & 0.36 & 0.24 \\
\hline $\mathrm{Cr}_{2} \mathrm{O}_{3}$ & 0.08 & 0.07 & 0.06 & 0.06 & 0.06 & 0.04 & 0.03 & 0.09 & 0.08 & 0.08 & 0.03 & 0.26 & 0.39 & 0.58 & 0.64 & 0.72 & 0.67 & 0.82 & 0.53 & 0.47 \\
\hline $\mathrm{FeO}^{*}$ & 3.75 & 4.92 & 5.88 & 7.32 & 5.46 & 5.73 & 4.98 & 4.31 & $\begin{array}{l}1.69 \\
1.69\end{array}$ & 1.87 & 3.92 & 3.75 & 3.12 & 4.19 & $\begin{array}{l}0.07 \\
3.72\end{array}$ & 3.62 & 2.96 & $\begin{array}{l}. .02 \\
4.06\end{array}$ & 4.31 & 3.59 \\
\hline MnO & $\begin{array}{l}0.65 \\
0.67\end{array}$ & $\begin{array}{l}4.92 \\
0.59\end{array}$ & $\begin{array}{l}3.00 \\
0.43\end{array}$ & 0.79 & $\begin{array}{l}3.40 \\
0.99\end{array}$ & 0.09 & $\begin{array}{l}4.90 \\
0.63\end{array}$ & $\begin{array}{l}4.31 \\
0.49\end{array}$ & $\begin{array}{l}1.19 \\
0.18\end{array}$ & $\begin{array}{l}1.07 \\
0.17\end{array}$ & 0.61 & 0.69 & $\begin{array}{l}3.12 \\
0.54\end{array}$ & $\begin{array}{l}4.199 \\
0.48\end{array}$ & 0.46 & $\begin{array}{l}3.22 \\
0.29\end{array}$ & $\begin{array}{l}2.90 \\
0.27\end{array}$ & $\begin{array}{l}4.00 \\
0.43\end{array}$ & $\begin{array}{l}4.51 \\
0.58\end{array}$ & 0.42 \\
\hline $\mathrm{MgO}$ & 52.51 & 51.66 & 52.19 & 51.94 & 52.14 & 50.30 & 52.56 & 52.71 & 55.22 & 55.92 & 51.36 & 52.72 & 52.13 & 51.66 & $\begin{array}{l}0.40 \\
52.92\end{array}$ & 51.17 & 52.46 & 52.33 & 52.12 & 52.62 \\
\hline $\mathrm{NiO}$ & 0.35 & 0.32 & 0.29 & 0.36 & 0.42 & 0.29 & 0.28 & 0.36 & 0.34 & 0.37 & 0.33 & 0.89 & 0.92 & 0.55 & 0.62 & 0.41 & $\begin{array}{l}0.45 \\
0.35\end{array}$ & 0.29 & 0.37 & 0.44 \\
\hline $\mathrm{CaO}$ & 0.01 & 0.01 & 0.02 & 0.02 & 0.03 & 0.01 & 0.01 & 0.01 & 0.01 & 0.02 & 0.02 & 0.04 & 0.05 & 0.06 & 0.03 & 0.02 & 0.03 & 0.05 & 0.07 & 0.09 \\
\hline $\mathrm{Na}_{2} \mathrm{O}$ & 0.01 & 0.01 & 0.01 & 0.02 & 0.03 & 0.03 & 0.02 & 0.03 & 0.06 & 0.05 & 0.02 & 0.03 & 0.02 & 0.03 & 0.03 & 0.03 & 0.02 & 0.06 & 0.04 & 0.07 \\
\hline $\mathrm{K}_{2} \mathrm{O}$ & 0.01 & 0.01 & 0.01 & 0.02 & 0.02 & 0.02 & 0.01 & 0.01 & $\begin{array}{l}0.02 \\
0.02\end{array}$ & 0.02 & 0.01 & 0.02 & 0.04 & 0.01 & 0.01 & 0.01 & 0.01 & 0.03 & $\begin{array}{l}0.02 \\
0.02\end{array}$ & 0.05 \\
\hline Total & 99.19 & 99.59 & 99.92 & 99.73 & 99.30 & $\begin{array}{l}0.02 \\
98.69\end{array}$ & 99.08 & 99.35 & 99.95 & 99.19 & 99.25 & 99.24 & $\begin{array}{l}0.04 \\
99.36\end{array}$ & 99.73 & 99.56 & 98.83 & 99.75 & 99.60 & 99.03 & 99.69 \\
\hline $\mathrm{Si}$ & 1.01 & 1.01 & 0.99 & 0.96 & 0.98 & 1.03 & 0.99 & 1.00 & 1.00 & 0.98 & 1.00 & 0.98 & $\begin{array}{l}1.00 \\
\text {. }\end{array}$ & 1.00 & 0.98 & 1.02 & 1.02 & 1.00 & 0.99 & 1.00 \\
\hline $\mathrm{Ti}$ & 0.00 & 0.00 & 0.00 & 0.00 & 0.00 & 0.00 & 0.00 & 0.00 & 0.00 & 0.00 & 0.00 & 0.00 & 0.00 & 0.00 & 0.00 & 0.00 & 0.00 & 0.00 & 0.00 & 0.00 \\
\hline Al & 0.00 & 0.00 & 0.00 & 0.00 & 0.00 & 0.00 & 0.00 & 0.00 & 0.00 & 0.00 & 0.01 & 0.01 & 0.01 & 0.02 & 0.02 & 0.01 & 0.01 & 0.00 & 0.01 & 0.01 \\
\hline $\mathrm{Cr}$ & 0.00 & 0.00 & 0.00 & 0.00 & 0.00 & 0.00 & 0.00 & 0.00 & 0.00 & 0.00 & 0.00 & 0.01 & 0.01 & 0.01 & 0.01 & 0.01 & 0.01 & 0.02 & 0.01 & 0.01 \\
\hline $\mathrm{Fe}(\mathrm{ii})$ & 0.08 & 0.10 & 0.12 & 0.15 & 0.11 & 0.12 & 0.10 & 0.09 & 0.03 & 0.04 & 0.08 & 0.08 & 0.06 & 0.08 & 0.08 & 0.07 & 0.06 & 0.08 & 0.09 & 0.07 \\
\hline Mn & 0.01 & 0.01 & 0.01 & 0.02 & 0.02 & 0.00 & 0.01 & 0.01 & 0.00 & 0.00 & 0.01 & 0.01 & 0.01 & 0.01 & 0.01 & 0.01 & 0.01 & 0.01 & 0.01 & 0.01 \\
\hline $\mathrm{Mg}$ & $\begin{array}{l}1.89 \\
\end{array}$ & 1.86 & 1.88 & 1.90 & 1.90 & 1.82 & 1.90 & 1.90 & 1.95 & 2.00 & 1.89 & 1.91 & 1.87 & 1.86 & 1.90 & 1.84 & 1.86 & 1.88 & 1.89 & 1.88 \\
\hline Ni & 0.01 & 0.01 & 0.01 & 0.01 & 0.01 & 0.01 & 0.01 & 0.01 & 0.01 & 0.01 & 0.01 & 0.02 & 0.02 & 0.01 & 0.01 & 0.01 & 0.01 & 0.01 & 0.01 & 0.01 \\
\hline $\mathrm{Ca}$ & 0.00 & 0.00 & 0.00 & 0.00 & 0.00 & 0.00 & 0.00 & 0.00 & 0.00 & 0.00 & 0.00 & 0.00 & 0.00 & 0.00 & 0.00 & 0.00 & 0.00 & 0.00 & 0.00 & $\begin{array}{l}0.01 \\
0.00\end{array}$ \\
\hline Fo & 95.48 & 94.35 & 93.64 & 91.94 & 93.50 & 93.91 & 94.35 & 95.13 & 98.13 & 98.00 & 95.28 & 95.48 & 96.20 & 95.17 & 95.75 & 95.89 & 96.66 & 95.40 & 94.99 & 95.90 \\
\hline $\mathrm{Fa}$ & 3.83 & 5.04 & 5.92 & 7.27 & 5.49 & 6.00 & 5.01 & 4.36 & $\begin{array}{l}90.10 \\
1.69\end{array}$ & 1.84 & 4.08 & 3.81 & 3.23 & 4.33 & 3.78 & 3.81 & $\begin{array}{l}0.00 \\
3.06\end{array}$ & $\begin{array}{l}3.40 \\
4.15\end{array}$ & 4.41 & 3.67 \\
\hline $\begin{array}{l}\mathrm{Ta} \\
\mathrm{Tp}\end{array}$ & $\begin{array}{l}0.00 \\
0.69\end{array}$ & 0.61 & 0.44 & 0.80 & 1.01 & $\begin{array}{l}0.09 \\
0.09\end{array}$ & 0.64 & $\begin{array}{l}4.000 \\
0.51\end{array}$ & 0.18 & $\begin{array}{l}1.84 \\
0.17\end{array}$ & $\begin{array}{l}4.08 \\
0.64\end{array}$ & $\begin{array}{l}3.81 \\
0.71\end{array}$ & $\begin{array}{l}3.23 \\
0.57\end{array}$ & $\begin{array}{l}4.33 \\
0.50\end{array}$ & $\begin{array}{l}3.18 \\
0.47\end{array}$ & $\begin{array}{l}3.81 \\
0.31\end{array}$ & $\begin{array}{l}3.00 \\
0.28\end{array}$ & $\begin{array}{l}4.15 \\
0.45\end{array}$ & $\begin{array}{l}4.41 \\
0.60\end{array}$ & $\begin{array}{l}3.07 \\
0.44\end{array}$ \\
\hline
\end{tabular}

Unfortunately, pyroxene minerals are observed only in serpentinized peridotites. Orthopyroxenes are typically enstatite (Supplementary Materials, Table S1), whilst clinopyroxenes (Supplementary materials Table S2) are classified as augites based on nomenclature [23] (Figure 4b). Orthopyroxenes contain (En 89.65-93.18, Fs 6.13-9.23, Wo 0.59-2.62) and $\mathrm{Mg \#}$ ranges from 89.65 to 93.18 , with a mean value of 91.03 , and low contents of $\mathrm{Al}_{2} \mathrm{O}_{3}$ and $\mathrm{TiO}_{2}$ (1.22-3.23; 0.01-0.04, respectively), similar to forearc peridotites [24]. Clinopyroxenes $\left(\mathrm{En}_{49.89-61.71}, \mathrm{Fs}_{1.94-5.33}, \mathrm{Wo}_{34.89-46}\right)$ contain $\mathrm{CaO}$ ranges from 17.87 to $23.14 \mathrm{wt} . \%$ and low $\mathrm{TiO}_{2}$ contents $(0.02-0.08 \mathrm{wt} . \%)$, reflecting their non-alkaline affinity [25]; in addition, they have $\mathrm{Al}_{2} \mathrm{O}_{3}$ contents of more than $1 \mathrm{wt} . \%$, suggesting their magmatic affinity [26]. Orthopyroxenes and clinopyroxenes have $\mathrm{Cr}_{2} \mathrm{O}_{3}$ less than $1 \mathrm{wt} . \%$, similar to those in forearc settings (Figure $4 \mathrm{c}, \mathrm{d}$ ). 

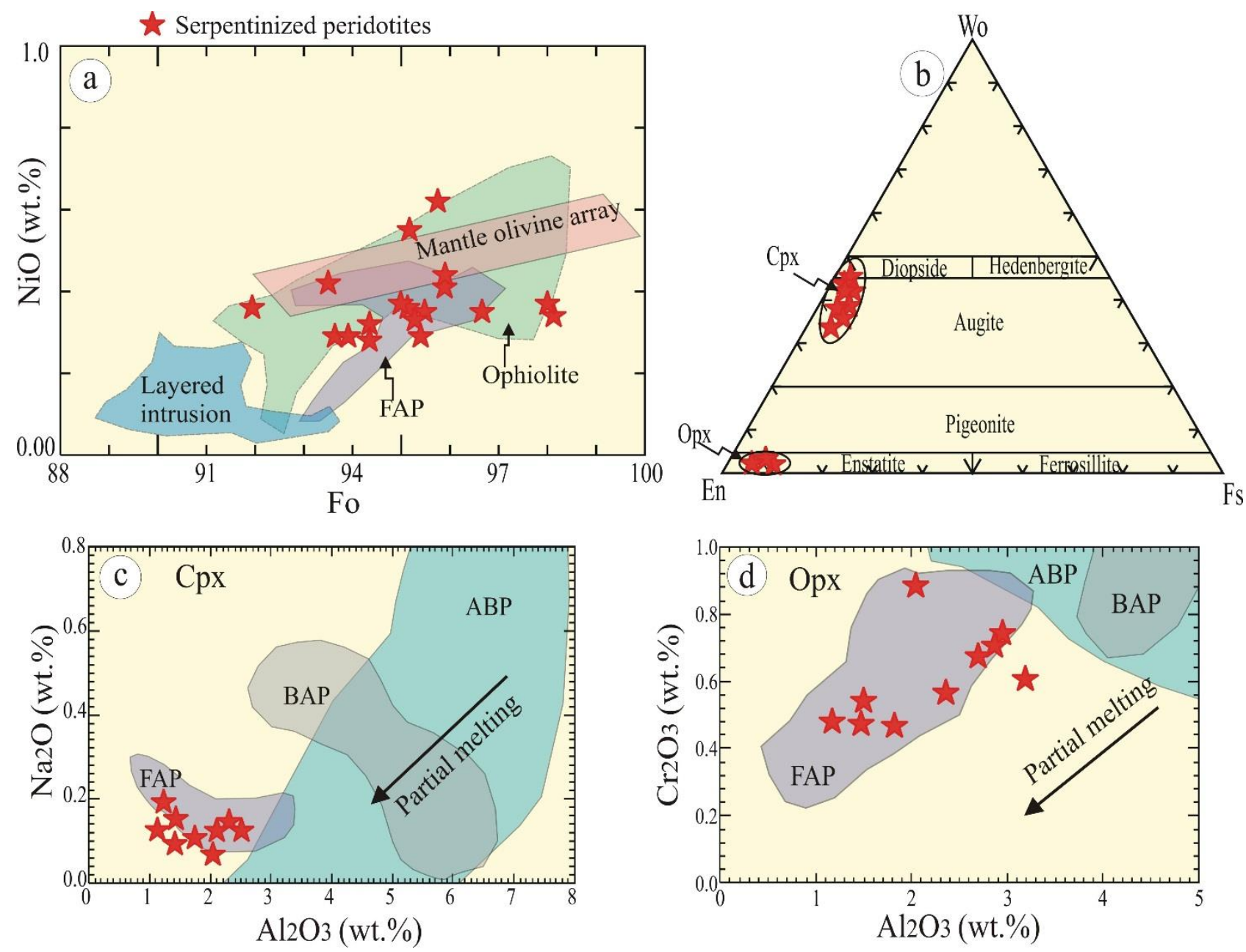

Figure 4. (a) Fo vs. NiO binary variation of olivines. Fields of olivine mantle array [27], ophiolite [22], forearc peridotite (FAP) [24], and layered intrusions [7]. (b) Enstatite (En)-ferrosilite (Fs)-wollastonite (Wo) nomenclature diagram of pyroxene minerals [23]. (c) $\mathrm{Na}_{2} \mathrm{O}$ vs. $\mathrm{Al}_{2} \mathrm{O}_{3}$ and (d) $\mathrm{Cr}_{2} \mathrm{O}_{3}$ vs. $\mathrm{Al}_{2} \mathrm{O}_{3}$ binary diagrams [24]. Field of forearc peridotite (FAP) and abyssal basin peridotite (ABP) obtained from [24], and back-arc peridotite (BAP) [28].

Spinel is predominantly composed of opaque minerals, and reveals heterogenetic composition; it can be distinguished into Cr-spinel (Supplementary Materials, Table S3) and Al-spinel (Supplementary Materials, Table S4). Al-spinel is found in the picotitic field, whilst Cr-spinel straddles the chromite field, and both are related to ophiolitic spinels $[29,30]$ (Figure 5a). Furthermore, Cr-spinel can be classified into chromite and magnesochromite, whereas Al-spinel is classified as spinel according to the $\mathrm{Cr} \#$ versus $\mathrm{Mg \#}$ binary discrimination diagram [31] (Figure 5b). Al-spinels contain high contents of $\mathrm{Al}_{2} \mathrm{O}_{3}$ (33.55-38.55, av. 35.77 wt.\%), $\mathrm{MgO}$ (12.37-16.12 av. 14.84 wt.\%), and $\mathrm{Mg} \#\left(\mathrm{Mg} / \mathrm{Mg}+\mathrm{Fe}^{2+}\right.$ ) (0.55-0.69, av. 0.64 wt.\%), and low contents of $\mathrm{FeO}\left(11.98-20.16\right.$, av. 16.66 wt.\%), $\mathrm{Cr}_{2} \mathrm{O}_{3}$ (28.48-34.17, av. 31.46 wt.\%), and $\mathrm{Cr} \#(\mathrm{Cr} /(\mathrm{Cr}+\mathrm{Al})(0.34-0.39$; av. 0.37$)$, compared to those of $\mathrm{Cr}$-spinel. In addition, they are found in the mantle array field, suggesting their magmatic nature (Figure 5c). The $\mathrm{Cr} \#$ contents of $\mathrm{Cr}$-spinel are similar to those of the Egyptian Eastern Desert [32]. Cr-spinels contain $\mathrm{YCr}\left(\mathrm{Cr} /\left(\mathrm{Cr}+\mathrm{Al}+\mathrm{Fe}^{+3}\right), \mathrm{YAl}\left(\mathrm{Al} /\left(\mathrm{Al}+\mathrm{Cr}+\mathrm{Fe}^{+3}\right)\right.\right.$ and $\mathrm{YFe}^{+3}$ $\left(\mathrm{Fe}^{+3} /\left(\mathrm{Fe}^{+3}+\mathrm{Al}+\mathrm{Cr}\right)\right.$, similar to forearc peridotites [32], whilst the contents of Al-spinel are similar to those of abyssal peridotites, which may be formed during inanition of subduction processes in proto-forearc environments (Figure $5 \mathrm{~d}$ ). 

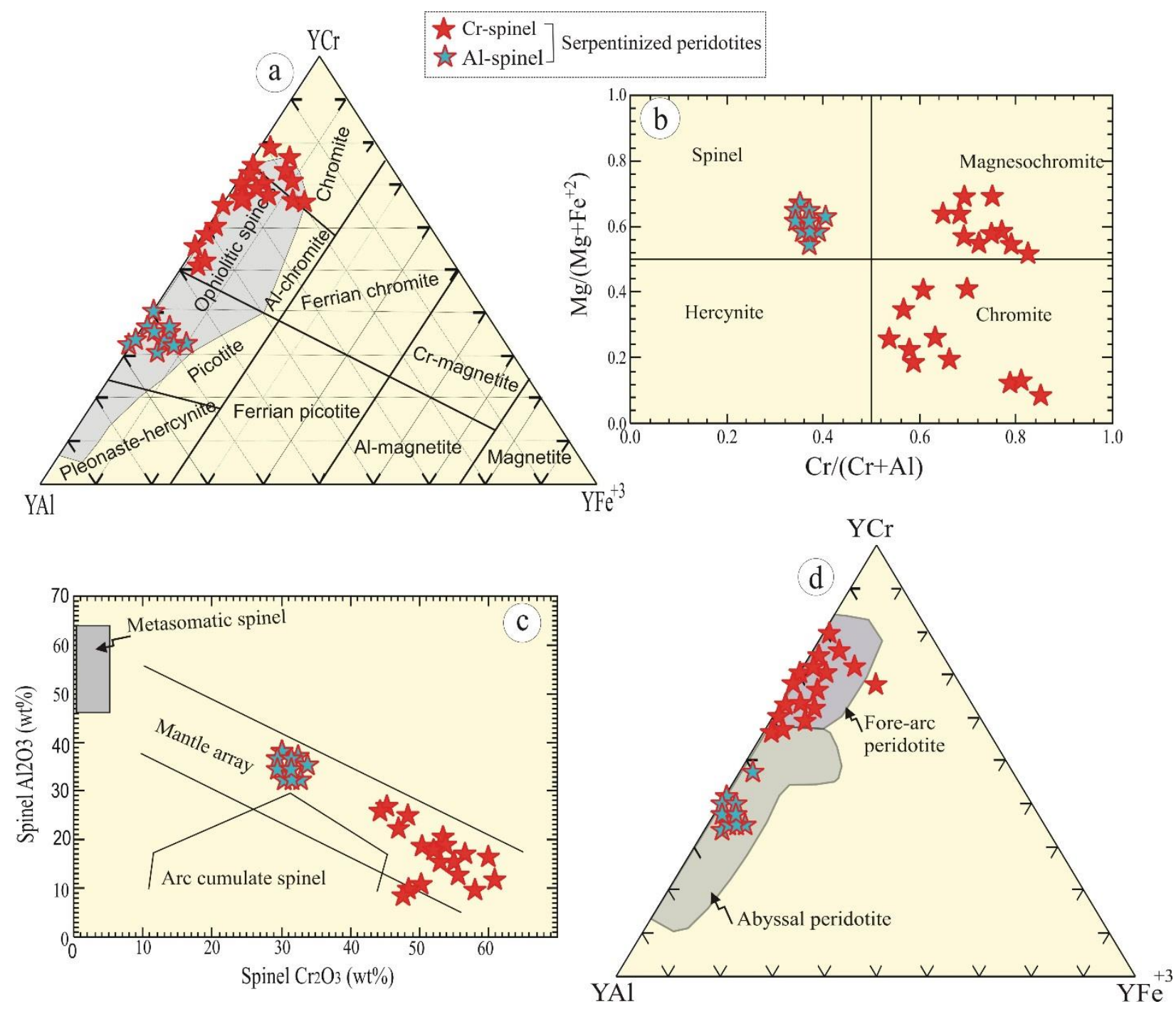

Figure 5. Spinel variation diagrams: (a) $\mathrm{Cr}-\mathrm{Al}-\mathrm{Fe}^{3+}$ ternary diagram $[29,30]$; (b) $\mathrm{Cr} /(\mathrm{Cr}+\mathrm{Al}) \mathrm{vs} . \mathrm{Mg} /\left(\mathrm{Mg}+\mathrm{Fe}^{2+}\right)[31]$; (c) $\mathrm{Cr}_{2} \mathrm{O}_{3}$ vs. $\mathrm{Al}_{2} \mathrm{O}_{3}$ binary diagram [32]; and (d) $\mathrm{Cr}-\mathrm{Al}-\mathrm{Fe}^{3+}$ ternary diagram [18].

EPMA results of serpentine minerals are shown in the Supplementary Materials (Table S5). Chrysotile exhibits low $\mathrm{Al}_{2} \mathrm{O}_{3}$ content (1.29-3.79, Figure 6a) and high $\mathrm{MgO}$ (43.36-47.35 wt.\%) and $\mathrm{SiO}_{2}(44.67-49.35 \mathrm{wt} . \%)$ relative to the antigorite phase in serpentinites (4.33-5.66 wt.\%; 41.69-45.55 wt.\%; 43.18-45.78 wt.\%, respectively) and serpentinized peridotites (4.55-5.44 wt.\%; 41.69-43.77 wt.\%; 43.79-45.89 wt.\%, respectively), which is consistent with the findings of [33] (Figure 6b-d). The chrysotile phase has a chemical affinity similar to that of olivine, whilst antigorite comes from orthopyroxene and olivine, reflecting a dunite protolith for the former, and both harzburgite and dunite for the latter phase (Figure 6e). The abundance of antigorite rather than chrysotile in the examined ultramafic rocks reflects prograde metamorphism [33,34].

Chlorite represents one of the main alteration products of the primary minerals (pyroxenes) in serpentinized peridotites. EPMA results show that the examined chlorites contain $\mathrm{SiO}_{2}$ ranges from 33.98 to $37.45 \mathrm{wt} . \%, \mathrm{Al}_{2} \mathrm{O}_{3}$ from 19.45 to $21.89 \mathrm{wt} \%, \mathrm{MgO}$ from 24.62 to $2.45 \mathrm{wt}$ \%, Si from 6.05 to $6.52 \mathrm{apfu}, \mathrm{Al}^{\mathrm{iv}}$ from 1.47 to $1.94 \mathrm{apfu}$, Fe\# from 0.22 to 0.28 , and Mg\# from 0.72 to 0.78 (Supplementary Materials, Table S6). On the Si (apfu) vs. $\mathrm{Fe}^{+2}+\mathrm{Fe}^{+3}$ (apfu) binary diagram [35], the examined chlorite is classified as pycnochlorite and diabantite (Figure 7a). $\mathrm{Al}^{\text {iv }}$ can be used to determine the chlorite temperature [36]. 
Using the equation in [37] $\left(\mathrm{T}=106.2^{*} \mathrm{Al}^{\mathrm{iv}}+17.5\right)$, the temperature of chlorite ranges from 174 to $224^{\circ} \mathrm{C}$.

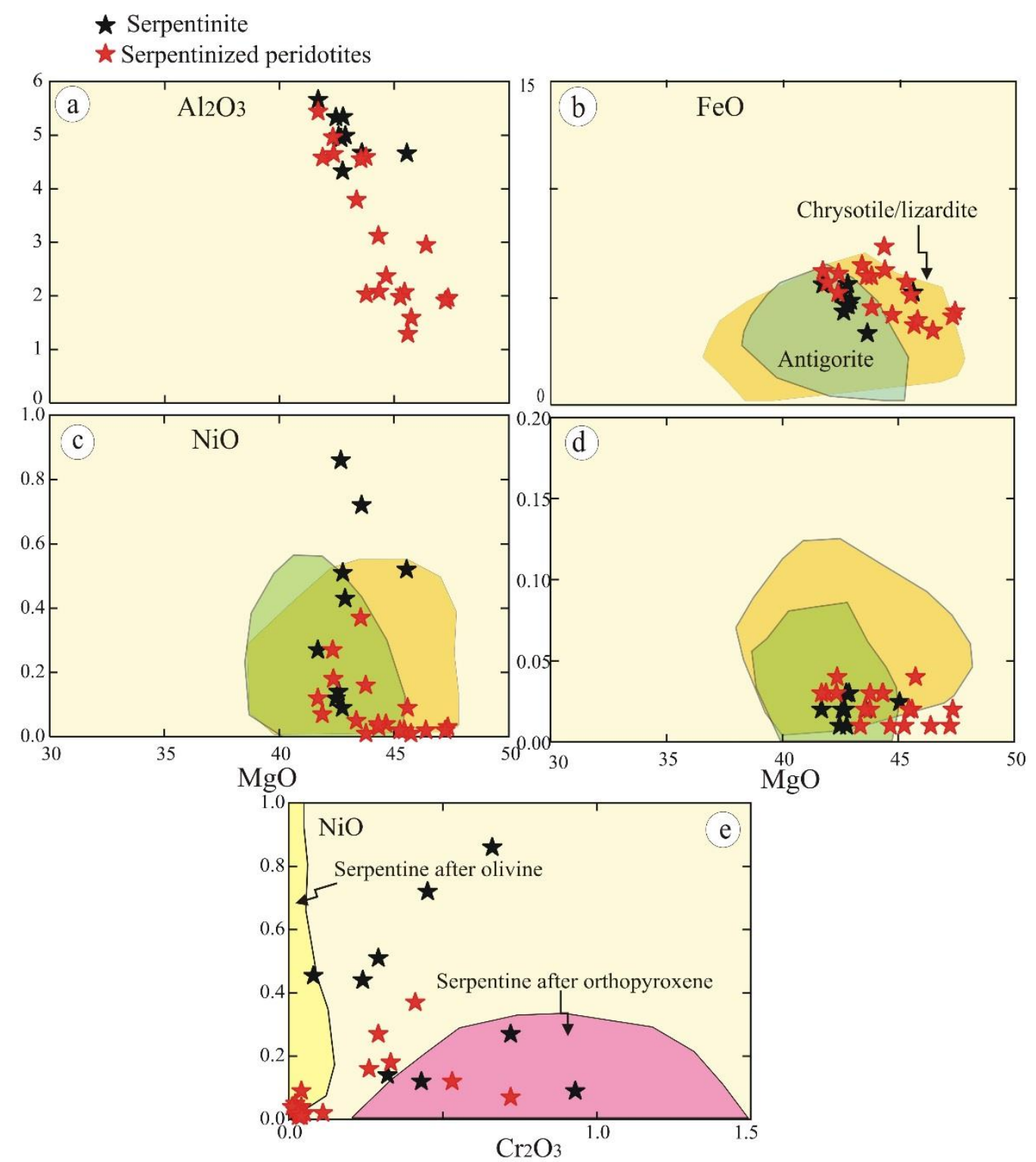

Figure 6. Serpentine binary variations: (a) $\mathrm{MgO}$ vs. $\mathrm{Al}_{2} \mathrm{O}_{3}$; (b) $\mathrm{MgO}$ vs. $\mathrm{FeO}$; (c) $\mathrm{MgO}$ vs. $\mathrm{NiO}$; (d) $\mathrm{MgO}$ vs. $\mathrm{MnO}$; and (e) $\mathrm{Cr}_{2} \mathrm{O}_{3}$ vs. NiO. Fields of antigorite and chrysotile are from [38]; serpentine after olivine and orthopyroxene fields are from [39].

Likewise, chlorite and tremolite represent the main alteration products of pyroxene. The examined tremolites (serpentinized peridotites) have $\mathrm{SiO}_{2}$ ranges from 48.34 to 59.81 wt. \%, $\mathrm{MgO}$ from 23.33 to $24.71 \mathrm{wt} . \%$, and $\mathrm{CaO}$ from 13.17 to $14.63 \mathrm{wt} . \%$ (Supplementary Materials, Table S7). They are classified as tremolite according to [40] (Figure 7b). They have $\mathrm{Ti}$ contents ranging from 0.003 to $0.014 \mathrm{apfu}$, with a mean value of $0.0036 \mathrm{apfu}$, reflecting their metamorphic nature. Furthermore, greenschist facies of the examined rocks are indicated by low $\mathrm{Na}$ and $\mathrm{K}$ contents of tremolite (av. $0.003 \mathrm{apfu}$ ) [41].

The investigated magnetites (serpentinites and serpentinized peridotites) were composed mainly of high $\mathrm{FeO}^{*}$ contents (av. 93.76), whilst their $\mathrm{TiO}_{2}$ (av. 0.055), $\mathrm{MnO}$ (av. 0.42), $\mathrm{CaO}$ (av. 0.017), $\mathrm{Na}_{2} \mathrm{O}$ (av. 0.027), $\mathrm{K}_{2} \mathrm{O}$ (av. 0.015), $\mathrm{Cr}_{2} \mathrm{O}_{3}$ (av. 0.29), $\mathrm{NiO}$ (av. 0.18), and $\mathrm{ZnO}$ (av. 0.031) contents are less than unity (Supplementary Materials, Table S8). They possess ulvospinel of less than $0.5 \mathrm{~mol} \%$, based on [42]. They are classified mainly as magnetites 
by using the $\mathrm{FeO}-\mathrm{TiO}_{2}-\mathrm{Fe}_{2} \mathrm{O}_{3}$ ternary diagram (Figure $7 \mathrm{c}$ ). In addition, the abundance of magnetites - either as patches, or filling fractures as veinlets-reflects the high degree of alteration of primary minerals, such as olivine [43].
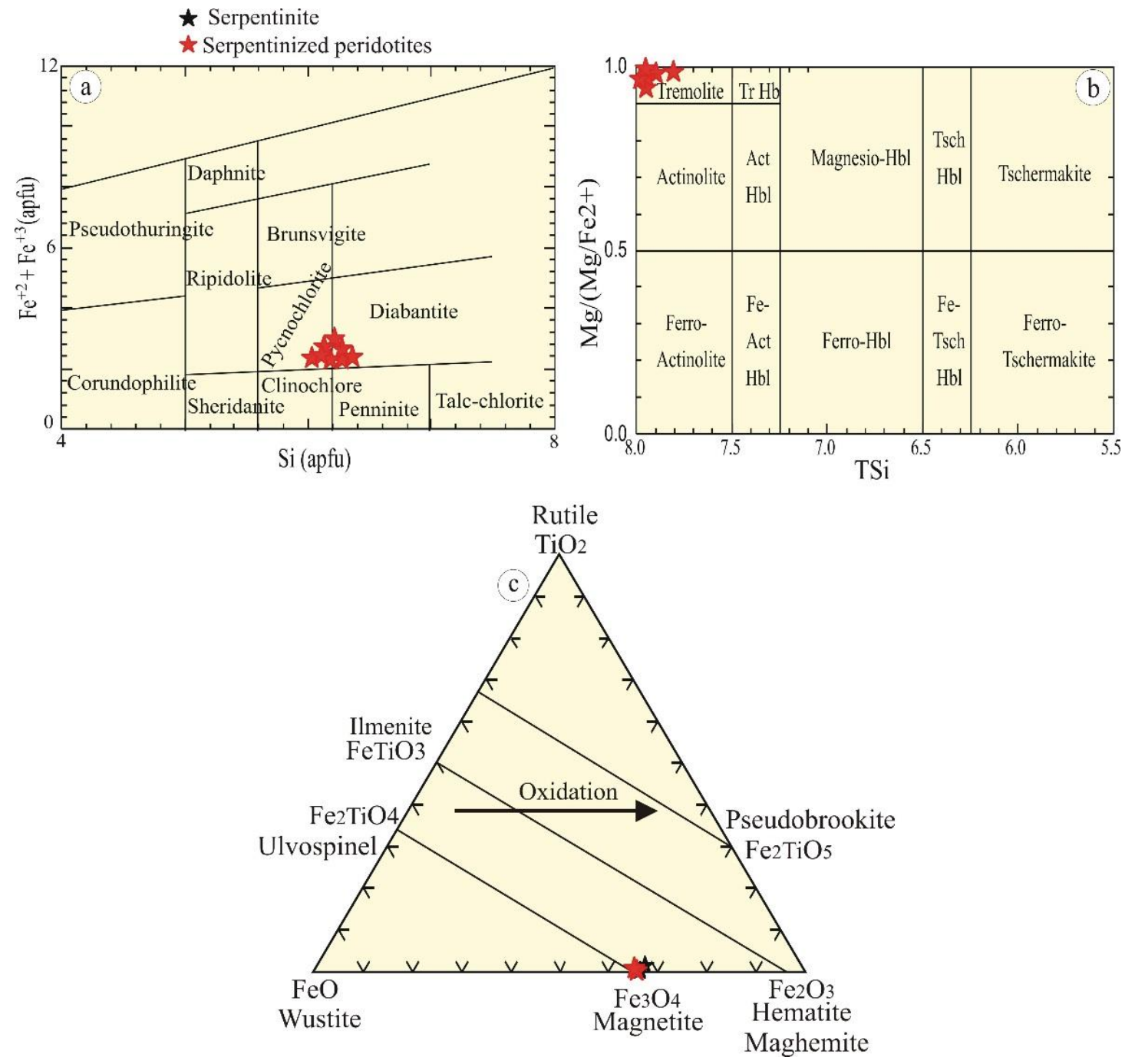

Figure 7. (a) Nomenclature diagram of chlorite from [35]; (b) TSi (apfu) vs. Mg\# discrimination diagram [40]; and (c) FeO$\mathrm{TiO}_{2}-\mathrm{Fe}_{2} \mathrm{O}_{3}$ discrimination diagram of magnetite and ilmenite.

EPMA of the examined talcs reveals that they consist mainly of $\mathrm{SiO}_{2}$ ranges from 56.35 to 60.99 wt. $\%, \mathrm{Na}_{2} \mathrm{O}$ from 12.77 to $15.44 \mathrm{wt} . \%, \mathrm{Al}_{2} \mathrm{O}_{3}$ from 18.47 to $23.45 \mathrm{wt} . \%, \mathrm{Mg \#}$ from 13.84 to 24.34 , K\# from 0.001 to 0.015 , and Fe\# from 75.65 to 86.16 (Supplementary Materials, Table S9). It is widely known that the talc forms as an alteration product after primary minerals (olivine and serpentine), suggesting high-temperature metamorphism.

\subsection{Whole-Rock Geochemistry}

The whole-rock (major, trace, and REEs) compositions of serpentinites and serpentinized peridotites are given in Table 2. Loss on ignition (LOI) varies from 5.27 to $11.5 \mathrm{wt} . \%$ for serpentinites, and from 4.16 to $10.7 \mathrm{wt} . \%$ for serpentinized peridotites, indicating a various and extensive degree of hydration and serpentinization. Therefore, bulk major oxides were recalculated to $100 \mathrm{wt} . \%$ based on an anhydrous to compensate for the variable serpentinization effect $[18,44]$. The average contents of major oxides in serpentinites and serpentinized peridotites reveal that the $\mathrm{TiO}_{2}, \mathrm{Al}_{2} \mathrm{O}_{3}, \mathrm{MnO}, \mathrm{Na}_{2} \mathrm{O}, \mathrm{K}_{2} \mathrm{O}$, and $\mathrm{P}_{2} \mathrm{O}_{5}$ contents are less than $1 \mathrm{wt} . \%$. In addition, the low $\mathrm{CaO}$ (av. 1.48 and $1.09 \mathrm{wt} . \%$, respectively) 
and $\mathrm{Al}_{2} \mathrm{O}_{3}$ (av. 0.76 and $0.59 \mathrm{wt} . \%$, respectively) contents of the examined serpentinites and serpentinized peridotites are similar to forearc peridotites and Pan-African serpentinites $[18,45]$ — especially those in the Egyptian Eastern Desert $[8,19,22]$ — suggesting their refractory origin [19] (Figure 8a,b).

Table 2. Abundances of major (wt.\%), trace, and rare earth elements (ppm) in ultramafic rocks.

\begin{tabular}{|c|c|c|c|c|c|c|c|c|c|c|c|c|}
\hline & \multicolumn{12}{|c|}{ Serpentinites } \\
\hline & IA1 & IA2 & IA3 & IA4 & IA5 & IA6 & IA7 & IA8 & IA9 & IA10 & IA11 & IA12 \\
\hline $\mathrm{SiO}_{2}$ & 43.10 & 42.02 & 42.18 & 43.57 & 41.63 & 41.14 & 43.45 & 42.66 & 40.64 & 43.06 & 40.93 & 42.09 \\
\hline $\mathrm{TiO}_{2}$ & 0.01 & 0.01 & 0.02 & 0.02 & 0.01 & 0.01 & 0.01 & 0.01 & 0.01 & 0.01 & 0.03 & 0.01 \\
\hline $\mathrm{Al}_{2} \mathrm{O}_{3}$ & 0.65 & 0.31 & 0.48 & 0.56 & 0.31 & 0.77 & 0.31 & 0.28 & 1.82 & 0.58 & 1.16 & 1.36 \\
\hline MgO & 44.72 & 43.44 & 44.10 & 44.30 & 44.86 & 44.73 & 45.36 & 46.36 & 46.39 & 43.65 & 42.94 & 42.77 \\
\hline $\mathrm{CaO}$ & 1.06 & 1.77 & 1.78 & 1.35 & 2.04 & 1.85 & 0.45 & 0.39 & 1.72 & 1.64 & 2.05 & 2.14 \\
\hline $\mathrm{MnO}$ & 0.10 & 0.12 & 0.09 & 0.08 & 0.14 & 0.13 & 0.10 & 0.16 & 0.14 & 0.15 & 0.14 & 0.09 \\
\hline $\mathrm{Fe}_{2} \mathrm{O}_{3}$ & 10.29 & 12.21 & 11.29 & 10.05 & 10.94 & 11.28 & 10.24 & 10.07 & 9.15 & 10.83 & 12.68 & 11.49 \\
\hline $\mathrm{Na}_{2} \mathrm{O}$ & 0.02 & 0.05 & 0.02 & 0.02 & 0.03 & 0.04 & 0.04 & 0.03 & 0.08 & 0.02 & 0.01 & 0.02 \\
\hline $\mathrm{K}_{2} \mathrm{O}$ & 0.02 & 0.02 & 0.01 & 0.01 & 0.01 & 0.02 & 0.01 & 0.02 & 0.01 & 0.03 & 0.04 & 0.01 \\
\hline $\mathrm{P}_{2} \mathrm{O}_{5}$ & 0.02 & 0.02 & 0.01 & 0.01 & 0.04 & 0.01 & 0.02 & 0.01 & 0.03 & 0.01 & 0.01 & 0.01 \\
\hline LOI & 9.10 & 8.40 & 11.50 & 10.21 & 10.80 & 9.30 & 5.27 & 9.10 & 8.10 & 8.70 & 8.23 & 8.40 \\
\hline $\mathrm{Ba}$ & 4.24 & 4.93 & 5.66 & 5.41 & 6.22 & 5.17 & 5.83 & 6.92 & 4.51 & 5.40 & 3.61 & 5.53 \\
\hline $\mathbf{R b}$ & 0.39 & 1.43 & 0.52 & 0.59 & 0.82 & 0.91 & 0.81 & 1.36 & 1.24 & 1.45 & 1.56 & 0.59 \\
\hline $\mathrm{Sr}$ & 5.91 & 4.84 & 4.56 & 2.39 & 2.87 & 2.97 & 3.81 & 3.52 & 3.11 & 3.32 & 2.91 & 2.66 \\
\hline Cs & 1.12 & 0.14 & 0.11 & 1.15 & 0.17 & 0.27 & 0.24 & 0.25 & 1.33 & 0.41 & 0.45 & 1.49 \\
\hline $\mathrm{Ga}$ & 1.96 & 0.82 & 0.75 & 0.86 & 1.02 & 1.09 & 1.13 & 1.79 & 0.82 & 0.84 & 1.63 & 1.48 \\
\hline $\mathrm{Ta}$ & 1.22 & 1.36 & 0.92 & 0.81 & 0.41 & 0.32 & 0.35 & 0.21 & 0.27 & 0.17 & 0.18 & 0.99 \\
\hline $\mathrm{Nb}$ & 3.12 & 2.24 & 2.19 & 1.27 & 0.37 & 1.41 & 0.49 & 2.15 & 2.09 & 1.72 & 1.65 & 1.15 \\
\hline Hf & 0.12 & 0.23 & 0.16 & 0.15 & 0.11 & 0.10 & 0.09 & 0.10 & 0.01 & 0.01 & 1.12 & 1.77 \\
\hline $\mathrm{Zr}$ & 2.75 & 1.32 & 1.09 & 1.02 & 2.59 & 0.52 & 1.75 & 0.87 & 2.12 & 1.29 & 1.15 & 1.25 \\
\hline$Y$ & 2.59 & 1.66 & 1.71 & 0.79 & 0.87 & 1.95 & 0.98 & 1.54 & 0.42 & 2.34 & 2.29 & 1.45 \\
\hline Th & 1.05 & 0.06 & 0.05 & 0.04 & 0.03 & 1.06 & 0.09 & 0.08 & 1.05 & 0.03 & 0.04 & 1.05 \\
\hline $\mathbf{U}$ & 0.02 & 0.01 & 0.01 & 0.01 & 0.02 & 0.05 & 0.01 & 0.02 & 0.08 & 0.01 & 0.01 & 0.08 \\
\hline $\mathrm{Ni}$ & 2459.00 & 2510.00 & 2422.00 & 1265.00 & 2456.00 & 2325.00 & 1382.00 & 2319.00 & 1566.00 & 1376.00 & 2435.00 & 1455.00 \\
\hline Co & 148.00 & 92.00 & 132.00 & 139.00 & 142.00 & 99.00 & 106.00 & 109.00 & 122.00 & 146.00 & 152.00 & 158.00 \\
\hline Sc & 17.00 & 18.00 & 10.00 & 17.00 & 8.00 & 9.00 & 5.00 & 14.00 & 16.00 & 10.00 & 11.00 & 9.00 \\
\hline V & 46.00 & 136.00 & 42.00 & 99.00 & 32.00 & 129.00 & 25.00 & 34.00 & 22.00 & 119.00 & 14.00 & 25.00 \\
\hline $\mathrm{Cu}$ & 15.00 & 28.00 & 14.00 & 19.00 & 38.00 & 17.00 & 25.00 & 32.00 & 30.00 & 15.00 & 12.00 & 13.00 \\
\hline $\mathrm{Pb}$ & 4.00 & 3.00 & 3.00 & 4.00 & 3.00 & 2.00 & 1.00 & 2.00 & 1.00 & 4.00 & 4.00 & 3.00 \\
\hline $\mathrm{Zn}$ & 66.00 & 46.00 & 39.00 & 45.00 & 52.00 & 62.00 & 71.00 & 79.00 & 82.00 & 33.00 & 30.00 & 49.00 \\
\hline $\mathrm{Bi}$ & 0.11 & 0.15 & 0.19 & 0.24 & 0.39 & 0.39 & 0.29 & 0.21 & 0.13 & 0.08 & 0.16 & 0.25 \\
\hline $\mathrm{Cd}$ & 0.20 & 0.20 & 0.40 & 0.20 & 0.20 & 0.30 & 0.10 & 0.10 & 0.30 & 0.10 & 0.20 & 0.20 \\
\hline Sn & 0.91 & 0.86 & 1.37 & 1.22 & 1.09 & 1.19 & 0.75 & 0.78 & 0.61 & 1.50 & 3.00 & 2.00 \\
\hline $\mathbf{W}$ & 1.30 & 1.75 & 1.62 & 1.35 & 1.50 & 1.77 & 1.31 & 0.97 & 0.86 & 1.39 & 1.45 & 0.75 \\
\hline Mo & 1.51 & 2.71 & 2.53 & 2.22 & 3.44 & 3.63 & 3.94 & 3.15 & 3.81 & 5.66 & 5.24 & 3.45 \\
\hline As & 1.88 & 0.75 & 0.68 & 0.91 & 0.04 & 0.05 & 0.09 & 0.76 & 0.89 & 1.09 & 1.15 & 1.12 \\
\hline $\mathrm{Sb}$ & 0.31 & 1.39 & 0.49 & 1.45 & 0.51 & 0.59 & 1.15 & 0.17 & 0.09 & 0.06 & 1.08 & 0.19 \\
\hline La & 0.65 & 0.58 & 1.44 & 0.16 & 0.12 & 0.79 & 0.66 & 0.15 & 0.13 & 0.88 & 0.56 & 1.27 \\
\hline $\mathrm{Ce}$ & 0.87 & 0.95 & 0.34 & 0.27 & 0.22 & 0.14 & 0.99 & 0.85 & 0.35 & 0.33 & 0.91 & 0.89 \\
\hline Pr & 0.21 & 0.32 & 0.27 & 0.39 & 0.45 & 0.35 & 0.66 & 0.59 & 0.32 & 0.35 & 0.11 & 0.42 \\
\hline Nd & 0.88 & 0.82 & 0.79 & 0.72 & 1.39 & 1.49 & 1.52 & 1.69 & 1.75 & 0.92 & 1.29 & 0.32 \\
\hline Sm & 0.39 & 0.36 & 0.45 & 0.56 & 0.47 & 0.58 & 0.51 & 0.33 & 0.37 & 0.27 & 0.42 & 0.37 \\
\hline Eu & 0.14 & 0.19 & 0.17 & 0.22 & 0.37 & 0.26 & 0.16 & 0.75 & 0.69 & 0.07 & 0.09 & 0.32 \\
\hline Gd & 0.52 & 0.45 & 0.49 & 0.59 & 0.69 & 0.28 & 0.32 & 0.35 & 0.46 & 0.51 & 0.53 & 0.41 \\
\hline $\mathrm{Tb}$ & 0.12 & 0.19 & 0.14 & 0.16 & 0.42 & 0.49 & 0.62 & 0.78 & 0.52 & 0.93 & 0.17 & 0.08 \\
\hline Dy & 0.57 & 0.63 & 0.69 & 0.61 & 0.15 & 0.99 & 0.37 & 0.15 & 0.31 & 0.02 & 0.19 & 0.22 \\
\hline Ho & 0.12 & 0.14 & 0.17 & 0.27 & 0.39 & 0.44 & 0.52 & 0.77 & 0.75 & 0.92 & 1.34 & 0.19 \\
\hline Er & 0.39 & 0.31 & 0.46 & 0.49 & 0.32 & 0.39 & 0.72 & 0.61 & 0.22 & 0.15 & 0.27 & 0.66 \\
\hline Tm & 0.08 & 0.09 & 0.12 & 0.19 & 0.29 & 0.45 & 0.35 & 0.52 & 0.59 & 0.74 & 0.79 & 0.81 \\
\hline $\mathrm{Yb}$ & 0.39 & 0.44 & 0.48 & 0.51 & 0.57 & 0.62 & 0.46 & 0.59 & 0.11 & 0.05 & 0.65 & 0.12 \\
\hline $\mathbf{L u}$ & 0.06 & 0.08 & 0.09 & 0.15 & 0.29 & 0.39 & 0.07 & 0.45 & 0.09 & 0.07 & 0.05 & 0.61 \\
\hline LREEs & 3.00 & 3.03 & 3.29 & 2.10 & 2.65 & 3.35 & 4.34 & 3.61 & 2.92 & 2.75 & 3.29 & 3.26 \\
\hline HREEs & 2.39 & 2.52 & 2.81 & 3.19 & 3.49 & 4.31 & 3.59 & 4.97 & 3.74 & 3.46 & 4.08 & 3.42 \\
\hline LREEs/HREEs & 1.26 & 1.20 & 1.17 & 0.66 & 0.76 & 0.78 & 1.21 & 0.73 & 0.78 & 0.79 & 0.81 & 0.95 \\
\hline$\sum$ REEs & 5.39 & 5.55 & 6.10 & 5.29 & 6.14 & 7.66 & 7.93 & 8.58 & 6.65 & 6.21 & 7.37 & 6.68 \\
\hline $\mathrm{Eu} / \mathrm{Eu}^{*}$ & 1.00 & 1.40 & 1.10 & 1.20 & 2.00 & 1.80 & 1.10 & 6.70 & 5.10 & 0.60 & 0.60 & 2.50 \\
\hline$(\mathrm{La} / \mathrm{Yb}) \mathrm{cn}$ & 1.10 & 0.90 & 2.00 & 0.20 & 0.10 & 0.90 & 1.00 & 0.20 & 0.80 & 11.90 & 0.60 & 7.20 \\
\hline$(\mathrm{Gd} / \mathrm{Yb}) \mathrm{cn}$ & 1.10 & 0.80 & 0.80 & 0.90 & 1.00 & 0.40 & 0.60 & 0.50 & 3.30 & 8.30 & 0.70 & 2.80 \\
\hline$(\mathrm{La} / \mathrm{Sm}) \mathrm{cn}$ & 1.00 & 1.00 & 2.00 & 0.20 & 0.20 & 0.90 & 0.80 & 0.30 & 0.20 & 2.10 & 0.80 & 2.20 \\
\hline
\end{tabular}


Table 2. Cont.

\begin{tabular}{|c|c|c|c|c|c|c|c|c|c|c|c|c|c|}
\hline & \multicolumn{13}{|c|}{ Serpentinized Peridotites } \\
\hline & AP1 & AP2 & AP3 & AP4 & AP5 & AP6 & AP7 & AP8 & AP9 & AP10 & AP11 & AP12 & AP13 \\
\hline $\mathrm{SiO}_{2}$ & 45.33 & 45.51 & 46.50 & 44.71 & 47.96 & 44.97 & 44.19 & 43.64 & 44.08 & 44.42 & 44.83 & 47.77 & 47.22 \\
\hline $\mathrm{TiO}_{2}$ & 0.01 & 0.01 & 0.01 & 0.01 & 0.03 & 0.01 & 0.01 & 0.01 & 0.01 & 0.01 & 0.01 & 0.01 & 0.01 \\
\hline $\mathrm{Al}_{2} \mathrm{O}_{3}$ & 0.55 & 0.82 & 0.71 & 0.38 & 1.27 & 0.67 & 0.79 & 0.44 & 0.20 & 0.42 & 0.39 & 0.30 & 0.49 \\
\hline MgO & 43.61 & 43.46 & 42.88 & 43.17 & 39.03 & 43.02 & 42.69 & 43.01 & 44.01 & 45.02 & 45.19 & 42.52 & 43.15 \\
\hline $\mathrm{CaO}$ & 2.05 & 1.52 & 1.42 & 1.24 & 0.02 & 0.96 & 1.49 & 0.75 & 0.65 & 0.93 & 0.95 & 0.99 & 1.35 \\
\hline $\mathrm{MnO}$ & 0.10 & 0.10 & 0.17 & 0.15 & 0.20 & 0.14 & 0.09 & 0.14 & 0.11 & 0.15 & 0.14 & 0.09 & 0.10 \\
\hline $\mathrm{Fe}_{2} \mathrm{O}_{3}$ & 8.28 & 8.53 & 8.25 & 10.24 & 11.43 & 10.13 & 10.61 & 11.93 & 10.85 & 9.00 & 8.43 & 8.26 & 7.61 \\
\hline $\mathrm{Na}_{2} \mathrm{O}$ & 0.03 & 0.02 & 0.02 & 0.04 & 0.02 & 0.05 & 0.07 & 0.05 & 0.06 & 0.03 & 0.01 & 0.01 & 0.02 \\
\hline $\mathrm{K}_{2} \mathrm{O}$ & 0.01 & 0.01 & 0.01 & 0.01 & 0.01 & 0.01 & 0.03 & 0.01 & 0.01 & 0.01 & 0.01 & 0.03 & 0.03 \\
\hline $\mathbf{P}_{2} \mathrm{O}_{5}$ & 0.02 & 0.01 & 0.02 & 0.03 & 0.02 & 0.02 & 0.02 & 0.01 & 0.01 & 0.01 & 0.03 & 0.02 & 0.01 \\
\hline LOI & 10.70 & 6.00 & 6.22 & 7.46 & 4.16 & 6.90 & 5.30 & 6.40 & 5.00 & 5.82 & 5.90 & 7.12 & 8.12 \\
\hline Ba & 9.70 & 10.00 & 9.00 & 5.00 & 6.00 & 3.00 & 4.00 & 4.00 & 5.00 & 4.00 & 10.00 & 6.00 & 5.00 \\
\hline $\mathbf{R b}$ & 0.67 & 1.12 & 0.19 & 0.25 & 1.75 & 1.85 & 0.49 & 1.22 & 0.27 & 0.35 & 0.27 & 0.33 & 1.40 \\
\hline $\mathrm{Sr}$ & 4.52 & 3.62 & 4.91 & 5.21 & 2.34 & 3.61 & 2.93 & 2.81 & 3.24 & 5.11 & 4.26 & 3.78 & 3.99 \\
\hline Cs & 0.41 & 0.19 & 0.11 & 0.13 & 0.22 & 0.32 & 0.18 & 0.27 & 0.36 & 0.14 & 0.28 & 0.37 & 0.49 \\
\hline Ga & 1.75 & 2.36 & 3.72 & 3.92 & 4.11 & 4.56 & 3.22 & 1.39 & 1.98 & 4.97 & 2.48 & 3.47 & 5.64 \\
\hline $\mathrm{Ta}$ & 1.22 & 1.36 & 0.86 & 0.55 & 0.45 & 0.36 & 0.24 & 0.26 & 0.22 & 0.33 & 0.44 & 0.66 & 0.99 \\
\hline $\mathrm{Nb}$ & 1.25 & 1.77 & 1.84 & 2.11 & 0.46 & 1.57 & 1.57 & 0.92 & 1.19 & 1.15 & 0.97 & 1.13 & 1.69 \\
\hline Hf & 0.91 & 1.25 & 1.19 & 0.87 & 0.15 & 0.11 & 0.13 & 1.22 & 1.45 & 1.69 & 1.89 & 1.25 & 1.10 \\
\hline $\mathrm{Zr}$ & 1.55 & 1.66 & 1.99 & 1.88 & 1.77 & 1.33 & 1.44 & 1.66 & 1.99 & 1.45 & 2.10 & 2.20 & 1.88 \\
\hline $\mathrm{Y}$ & 2.69 & 1.89 & 2.99 & 2.78 & 2.56 & 2.36 & 2.45 & 0.88 & 0.99 & 0.87 & 0.69 & 0.74 & 0.55 \\
\hline Th & 0.09 & 0.08 & 0.07 & 0.06 & 0.11 & 0.15 & 0.19 & 0.22 & 0.07 & 0.05 & 0.07 & 0.08 & 0.09 \\
\hline $\mathbf{U}$ & 0.02 & 0.01 & 0.02 & 0.03 & 0.02 & 0.01 & 0.02 & 0.02 & 0.01 & 0.04 & 0.03 & 0.04 & 0.02 \\
\hline $\mathrm{Ni}$ & 1590.0 & 1756.0 & 1816.0 & 1975.0 & 2632.0 & 1453.0 & 1415.0 & 1356.0 & 1466.0 & 2341.0 & 2566.0 & 1586.0 & 1439.0 \\
\hline Co & 99.00 & 133.00 & 170.00 & 122.00 & 109.00 & 95.00 & 87.00 & 76.00 & 132.00 & 145.00 & 121.00 & 102.00 & 75.00 \\
\hline Sc & 10.00 & 11.00 & 6.00 & 5.00 & 22.00 & 6.00 & 9.00 & 7.00 & 14.00 & 22.00 & 5.00 & 4.00 & 9.00 \\
\hline V & 49.00 & 69.00 & 75.00 & 99.00 & 106.00 & 110.00 & 59.00 & 67.00 & 84.00 & 93.00 & 87.00 & 118.00 & 37.00 \\
\hline $\mathrm{Cu}$ & 10.00 & 24.00 & 35.00 & 49.00 & 57.00 & 22.00 & 10.00 & 92.00 & 14.00 & 19.00 & 35.00 & 66.00 & 42.00 \\
\hline $\mathrm{Pb}$ & 2.00 & 4.00 & 6.00 & 2.00 & 10.00 & 12.00 & 11.00 & 3.00 & 7.00 & 5.00 & 6.00 & 4.00 & 4.00 \\
\hline $\mathrm{Zn}$ & 34.00 & 28.00 & 45.00 & 64.00 & 49.00 & 24.00 & 72.00 & 69.00 & 86.00 & 91.00 & 40.00 & 39.00 & 54.00 \\
\hline $\mathrm{Bi}$ & 0.36 & 0.35 & 0.24 & 0.11 & 0.36 & 0.58 & 0.45 & 0.16 & 0.13 & 0.69 & 0.45 & 0.36 & 0.78 \\
\hline $\mathrm{Cd}$ & 0.10 & 0.20 & 0.30 & 0.50 & 0.60 & 0.10 & 0.20 & 0.30 & 0.50 & 0.60 & 0.80 & 0.20 & 0.40 \\
\hline Sn & 1.00 & 2.00 & 1.00 & 2.00 & 2.00 & 1.00 & 1.00 & 1.00 & 1.00 & 2.00 & 1.00 & 2.00 & 2.00 \\
\hline $\mathbf{W}$ & 1.55 & 1.99 & 1.66 & 1.45 & 1.33 & 1.69 & 1.75 & 1.56 & 1.44 & 1.22 & 1.66 & 1.88 & 1.48 \\
\hline Mo & 2.60 & 4.20 & 4.90 & 5.60 & 3.40 & 1.50 & 1.80 & 1.40 & 2.30 & 4.20 & 6.20 & 5.70 & 3.10 \\
\hline As & 1.23 & 1.09 & 0.66 & 0.78 & 0.34 & 0.56 & 0.78 & 1.59 & 1.89 & 2.45 & 2.46 & 2.15 & 2.14 \\
\hline $\mathrm{Sb}$ & 0.12 & 0.14 & 0.47 & 0.39 & 1.58 & 1.26 & 1.11 & 1.10 & 0.78 & 0.78 & 0.65 & 0.73 & 0.42 \\
\hline La & 0.77 & 0.69 & 0.89 & 0.45 & 0.36 & 0.11 & 0.45 & 0.45 & 0.78 & 0.66 & 0.88 & 0.99 & 0.77 \\
\hline $\mathrm{Ce}$ & 0.99 & 0.68 & 0.45 & 0.47 & 0.78 & 0.66 & 0.78 & 0.86 & 0.65 & 0.78 & 0.48 & 0.78 & 0.89 \\
\hline Pr & 0.66 & 0.28 & 0.32 & 0.77 & 0.84 & 1.24 & 1.35 & 0.37 & 0.45 & 0.92 & 0.75 & 1.55 & 0.69 \\
\hline Nd & 0.12 & 0.09 & 0.07 & 0.08 & 0.05 & 0.52 & 0.65 & 0.73 & 0.09 & 0.24 & 0.45 & 0.98 & 1.48 \\
\hline Sm & 0.25 & 0.29 & 0.38 & 0.69 & 0.75 & 0.45 & 0.51 & 0.24 & 0.55 & 0.66 & 0.66 & 0.77 & 0.89 \\
\hline $\mathrm{Eu}$ & 0.46 & 0.29 & 0.27 & 0.05 & 0.14 & 0.29 & 0.87 & 0.69 & 0.34 & 0.23 & 0.14 & 0.11 & 0.08 \\
\hline Gd & 0.03 & 0.02 & 0.14 & 0.05 & 0.04 & 0.15 & 0.18 & 0.27 & 0.32 & 0.42 & 0.56 & 0.88 & 0.64 \\
\hline $\mathrm{Tb}$ & 0.27 & 0.05 & 0.03 & 0.08 & 0.02 & 0.04 & 0.03 & 0.28 & 0.68 & 0.44 & 0.33 & 0.11 & 0.28 \\
\hline Dy & 0.03 & 0.19 & 0.14 & 0.13 & 0.23 & 0.35 & 0.29 & 0.15 & 0.44 & 0.19 & 0.36 & 0.45 & 0.56 \\
\hline Ho & 0.17 & 0.26 & 0.19 & 0.62 & 0.22 & 0.07 & 0.05 & 0.14 & 0.66 & 0.79 & 0.69 & 0.84 & 0.46 \\
\hline Er & 0.14 & 0.06 & 0.13 & 0.02 & 0.07 & 0.16 & 0.32 & 0.15 & 0.24 & 0.17 & 0.36 & 1.26 & 0.45 \\
\hline Tm & 1.06 & 0.05 & 0.04 & 1.03 & 0.12 & 0.14 & 1.06 & 0.03 & 0.04 & 0.05 & 0.36 & 0.45 & 0.22 \\
\hline $\mathrm{Yb}$ & 0.12 & 0.04 & 0.03 & 0.11 & 0.02 & 0.05 & 0.06 & 0.03 & 0.13 & 0.22 & 0.14 & 0.58 & 0.69 \\
\hline Lu & 0.11 & 0.12 & 0.13 & 0.24 & 0.06 & 0.18 & 0.15 & 0.08 & 0.07 & 0.14 & 0.15 & 0.22 & 0.36 \\
\hline LREEs & 2.79 & 2.03 & 2.11 & 2.46 & 2.78 & 2.98 & 3.74 & 2.65 & 2.52 & 3.26 & 3.22 & 5.07 & 4.72 \\
\hline HREES & 2.39 & 1.08 & 1.10 & 2.33 & 0.92 & 1.43 & 3.01 & 1.82 & 2.92 & 2.65 & 3.09 & 4.90 & 3.74 \\
\hline LREEs/HREEs & 1.17 & 1.87 & 1.92 & 1.05 & 3.02 & 2.08 & 1.24 & 1.46 & 0.86 & 1.23 & 1.04 & 1.03 & 1.26 \\
\hline$\sum$ REEs & 5.18 & 3.11 & 3.21 & 4.79 & 3.70 & 4.41 & 6.75 & 4.47 & 5.44 & 5.91 & 6.31 & 9.97 & 8.46 \\
\hline $\mathrm{Eu} / \mathrm{Eu}^{*}$ & 9.00 & 5.00 & 3.00 & 0.40 & 1.00 & 2.70 & 7.20 & 8.30 & 2.30 & 1.30 & 0.70 & 0.40 & 0.30 \\
\hline$(\mathrm{La} / \mathrm{Yb}) \mathrm{cn}$ & 4.30 & 11.70 & 20.00 & 2.80 & 12.20 & 1.50 & 5.10 & 10.10 & 4.10 & 2.00 & 4.20 & 1.20 & 0.80 \\
\hline$(\mathrm{Gd} / \mathrm{Yb}) \mathrm{cn}$ & 0.20 & 0.40 & 3.80 & 0.40 & 1.60 & 2.40 & 2.40 & 7.30 & 2.00 & 1.50 & 3.20 & 1.20 & 0.70 \\
\hline$(\mathrm{La} / \mathrm{Sm}) \mathrm{cn}$ & 1.90 & 1.50 & 1.50 & 0.40 & 0.30 & 0.20 & 0.60 & 1.20 & 0.90 & 0.60 & 0.80 & 0.80 & 0.50 \\
\hline
\end{tabular}

The $\mathrm{SiO}_{2} / \mathrm{MgO}$ ratio of the studied rocks ranges from 0.87 to 1.22 , similar to the ophiolitic peridotites in [45] (Figure 8c). In terms of the $\mathrm{CaO}-\mathrm{Al}_{2} \mathrm{O}_{3}-\mathrm{MgO}$ ternary diagram, the studied rocks are located in the dunite field, with a few samples in harzburgite (Figure 8d). Lack of alteration of clinopyroxene is associated with dissolution of $\mathrm{Ca}$ [27] in the examined rocks; therefore, they have very low $\mathrm{CaO}$ contents, below the values of the depleted (DM, 3.5 wt.\%) [46] and primitive mantle (PM, 3.7 wt.\%) [47]. Furthermore, the $\mathrm{Al}_{2} \mathrm{O}_{3}$ contents of the studied rocks are less than that of the $\mathrm{PM}\left(\mathrm{Al}_{2} \mathrm{O}_{3}\right.$ ca. $4.5 \mathrm{wt}$ \%) [47]. Likewise, the average $\mathrm{MgO}$ content is high relative to $\mathrm{PM}$ composition, suggesting that the mantle melting is more than $20 \%$. Conversely, $\mathrm{SiO}_{2}$ and $\mathrm{Fe}_{2} \mathrm{O}_{3}$ concentrations are lower than the values of the PM. 

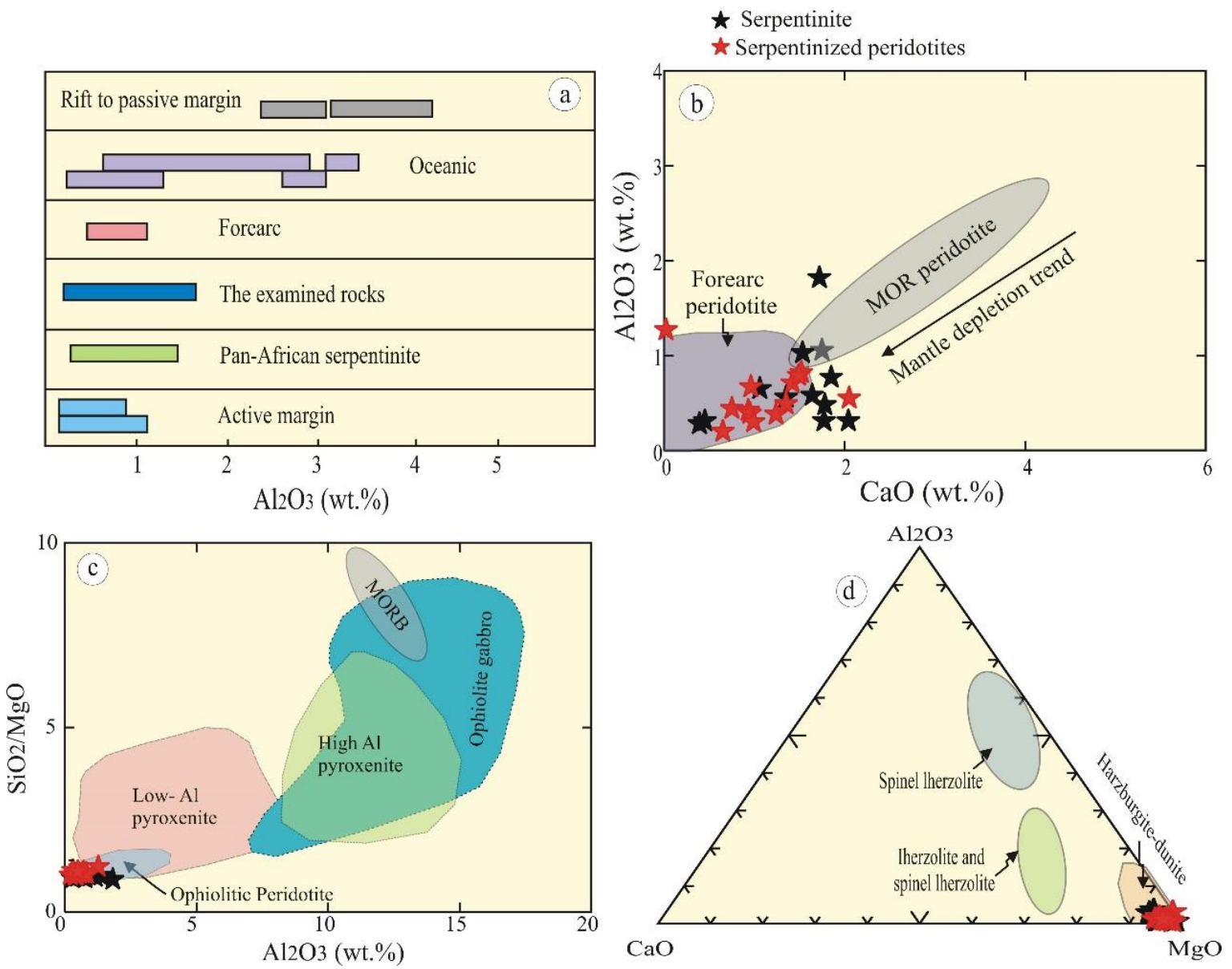

Figure 8. (a) $\mathrm{Al}_{2} \mathrm{O}_{3}$ of the examined rocks compared to Pan-African serpentinites [22] and others from [48]; (b) CaO vs. $\mathrm{Al}_{2} \mathrm{O}_{3}$ of bulk rock [49]; (c) $\mathrm{Al}_{2} \mathrm{O}_{3}$ vs. $\mathrm{SiO}_{2} / \mathrm{MgO}$ diagram [45]; and (d) $\mathrm{CaO}-\mathrm{Al}_{2} \mathrm{O}_{3}-\mathrm{MgO}$ ternary diagram [50].

The incompatible trace elements of the examined mantle rocks are depleted in highfield-strength elements (HFSEs)—such as Zr (0.52-2.75 ppm), Hf (0.0.1-1.89 ppm), Nb (0.37$3.12 \mathrm{ppm})$, and Ta (0.17-1.36 ppm) —and low-field-strength elements (LFSEs), including $\mathrm{Rb}(0.19-1.85 \mathrm{ppm}), \mathrm{Ba}(3-10 \mathrm{ppm})$, and Sr (2.34-5.91 ppm) (Table 2). Multiple elements of the primitive mantle (PM)-normalized diagram [51] (Figure 9a) show a strong negative Ta anomaly and slight enrichment of LFSEs relative to HFSEs, which are consistent with SSZ geochemical affinity [52]. Furthermore, they reveal a slightly positive $\mathrm{Pb}$ anomaly, similar to forearc peridotites [44]. This may be related to the effects of percolation of fluid during serpentinization processes [18]. They also possess high concentrations of transition elements, such as Ni and Co. Ni (1265-2510, av. 1982 ppm) and Co (92-158, av. 128 ppm) contents are higher in serpentinites than in serpentinized peridotites (Ni, av. 1825; av. Co, $114 \mathrm{ppm})$. Wide variation of $\mathrm{Ni}$ may be related to the dissemination of $\mathrm{Ni}$ in ferromagnesian minerals by serpentinization processes [53]. In addition, $\mathrm{Ni}^{2+}$ substitutes $\mathrm{Mg}^{2+}$ in olivine, and holds with $\mathrm{Mg}^{2+}$ even during and after serpentinization processes. 

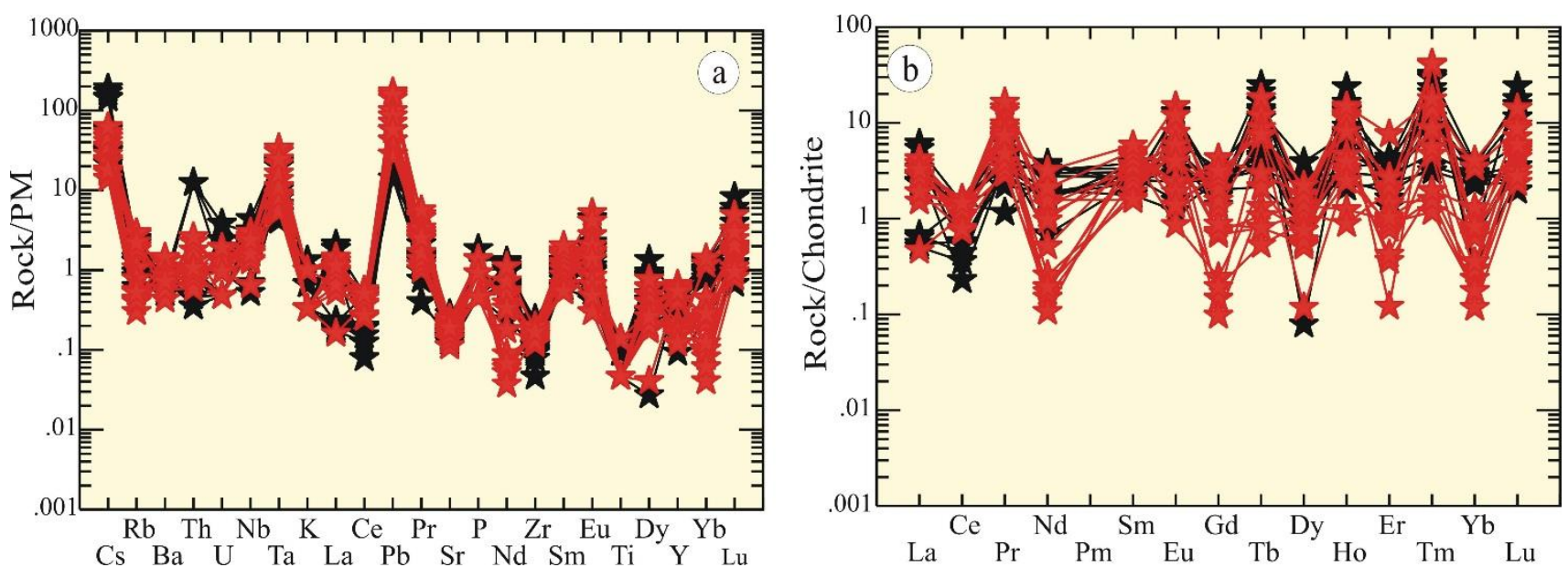

Figure 9. (a) Trace elements of the examined ultramafics normalized to the primitive mantle; and (b) chondrite-normalized rare earth elements [51].

Furthermore, we noticed a narrow range of Co contents, which may be related to the substitution by $\mathrm{Fe}^{+2}$-due to the similarity of ionic radii-and $\mathrm{Mg}^{+2}$ [53]. REEs are variable even in the same rock units, and reveal zigzag patterns (Figure $9 \mathrm{~b}$ ). The examined serpentinites are depleted in REEs (av. $\sum$ REEs $=6.6 \mathrm{ppm}$ ), since their light rare earth element (LREE) contents range from 0.2 to 2.2, and their heavy rare earth element (HREE) contents range from 0.5 to 8.3 [51]. Likewise, the examined serpentinized peridotites contain $\sum$ REEs ranging from 3.11 to $9.97 \mathrm{ppm}$, and slightly less fractionated LREEs $\left((\mathrm{La} / \mathrm{Sm})_{\mathrm{N}}=0.2-1.9\right)$ relative to HREEs $\left((\mathrm{Gd} / \mathrm{Yb})_{\mathrm{N}}=0.2-7.3\right)$, which is consistent with forearc serpentinites [18].

\section{Discussion}

\subsection{Protolith Changes and Element Mobility}

Slight changes in the bulk major oxides are estimated from the $\left(\mathrm{TiO}_{2}+\mathrm{MgO}+\mathrm{Al}_{2} \mathrm{O}_{3}\right.$ $\left.+\mathrm{Fe}_{2} \mathrm{O}_{3}+\mathrm{CaO}+\mathrm{MnO}+\mathrm{K}_{2} \mathrm{O}+\mathrm{Na}_{2} \mathrm{O}+\mathrm{P}_{2} \mathrm{O}_{5}\right) / \mathrm{SiO}_{2}$ ratios [18]. The calculated sum oxides $/ \mathrm{SiO}_{2}$ ratio (1.08-1.46) is similar to those of [54]. The investigated rocks represent some of the metamorphosed Egyptian ultramafic rocks with variable amounts of serpentine, tremolite, and talc, and fewer chlorite minerals. The presence of antigorite and talc suggest high temperature [55]. REEs and HFS elements are considered immobile, especially during alteration, in comparison with LFSEs — such as $\mathrm{Cs}, \mathrm{Rb}, \mathrm{K}, \mathrm{Sr}$, and U—even during low alteration [18]. Serpentinites represent as an alteration products of low temperature of ultramafic rocks, making some changes of the protolith chemical composition by water addition $[3,18,56]$. In addition, some major oxides—such as $\mathrm{CaO}$ and $\mathrm{SiO}_{2}[49]$ - can be remobilized during serpentinization processes. According to [49], the completely serpentinite rocks are more depleted in $\mathrm{CaO}$ relative to partially serpentinized rocks. Some physical properties of the protolith — such as density and magnetism —are also changed by serpentinization processes. Protoliths have higher density and lower magnetism than serpentinites [56], due to magnetite formation by serpentinization, as represented by the following equation:

$$
(\mathrm{Mg}, \mathrm{Fe})_{2} \mathrm{SiO}_{4}+\mathrm{H}_{2} \mathrm{O} \rightarrow(\mathrm{Mg}, \mathrm{Fe})_{3} \mathrm{Si}_{2} \mathrm{O}_{5}(\mathrm{OH})_{4}+\mathrm{Fe}_{3} \mathrm{O}_{4}+(\mathrm{Mg}, \mathrm{Fe})(\mathrm{OH})_{2}+\mathrm{H}_{2}
$$

There are two types of serpentinites based on the major serpentine minerals: (1) chrysotile is formed by retrograde metamorphism of primary minerals (olivine, orthopyroxene, and clinopyroxene) at low temperatures $\left(<300^{\circ} \mathrm{C}\right)$, and (2) antigorite serpentinites are formed by prograde metamorphism of lizardite and chrysotile at temperature ranges from $320^{\circ} \mathrm{C}$ and $390^{\circ} \mathrm{C}$, and become stable above $390^{\circ} \mathrm{C}$ [56]. Lizardite represents the most common serpentine mineral, whereas chrysotile is the least common [34]. They crystallize at identical temperature and pressure, but in different modes, where chrysotile precipitates a void 
space from a liquid state, whilst lizardite precipitates from a solid state on the surface of olivine [56]. Transition of lizardite or chrysotile to antigorite within water-saturated open systems takes place according to the following equation:

$$
\text { Lizardite (Chrysotile) }+\mathrm{SiO}_{2}\left(\text { aq) } \rightarrow \text { antigorite }+\mathrm{H}_{2} \mathrm{O}\right.
$$

Major oxides $\left(\mathrm{Al}_{2} \mathrm{O}_{3}\right)$ and some trace elements-especially HFSEs (e.g., $\mathrm{Zr}, \mathrm{Th}, \mathrm{Nb}$, Hf) - of serpentinites and serpentinized peridotites exhibit little change, tending to be immobile during the different metamorphic conditions, whilst LFSEs are expected to be mobile [13,57]. Fluid mobile elements (FMEs), encompasses LFSEs (Rb, Sr, Ba, U \& Cs) and semi-volatile elements (e.g., $\mathrm{Pb}$ ) are enriched in serpentinites relative to other of the same compatibility either serpentinites developed at supra-subduction or MOR [29]—due to high concentrations of these elements as a result of fluid-rock interaction leverage [17].

Serpentinization of mantle rocks is achieved via the formation of serpentine minerals and the addition of $\mathrm{H}_{2} \mathrm{O}$ at theoretical contents of up to $12.38 \mathrm{wt} . \% \pm 2.99 \mathrm{wt} . \%$ [18]. For this reason, the degree of serpentinization can be assessed by LOI. According to [57], samples with LOI of more than $6 \mathrm{wt}$ \% are considered to be altered. LOI reached up to $11.5 \mathrm{wt} . \%$ in the studied serpentinites, and $10.7 \mathrm{wt} . \%$ in serpentinized peridotites, supporting the role of hydrothermal alteration. There was a varying degree of serpentinization processes in all samples, where LOI varied from 4.16 to $11.5 \mathrm{wt}$ \% , reflecting moderately to completely serpentinized samples.

\subsection{Partial Melting Processes}

Whole-rock geochemistry and mineral chemistry of primary mantle minerals can be used to manifest different processes affecting the protolith, including partial melting and metasomatism [44,58]. It is noticeable that the examined rocks contain low concentrations of $\mathrm{TiO}_{2}$, ranging from 0.01 to $0.03 \mathrm{wt}$ \%, suggesting a high degree of partial melting [59].

On the other hand, systematic changes in primary minerals (olivine, pyroxene, and spinel) can be used as a key to deduce historical processes such as metasomatism and melting degree [60]. It is noteworthy that $\mathrm{Cr}$-spinels are more resistant primary minerals and can be used to infer the degree of partial melting [60]. The examined Cr-spinels of

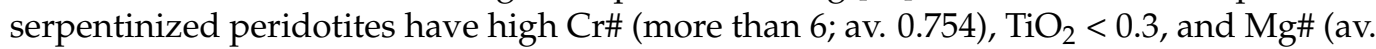
$0.42)$, reflecting a high degree of partial melting (>35\%) (Figure 10a) that developed in a forearc setting [22,38,61,62]. In terms of $\mathrm{Cr} \#$ vs. $\mathrm{TiO}_{2}$ in $\mathrm{Cr}$-spinel, they exhibit depleted mantle, and the examined sample was located in the fresh $\mathrm{Cr}$-spinel of the Egyptian Eastern Desert and forearc fields (Figure 10b).

The Cr\# content of both spinel types (especially Cr-rich) can be used to detect the degree of partial melting in the host rocks [62]. The degree of partial melting in spinel mantle can be calculated using the equation described in [60] $(\mathrm{F}=10 \times \mathrm{nCr} \#+24)$, where $\mathrm{F}$ is the melting degree (wt.\%). Based on this empirical equation, the examined rocks had experienced partial melting degrees ranging from 13.32 to $14.63 \%$ for Al-spinel, and from 17.34 to $24 \%$ for $\mathrm{Cr}$-spinel, which is consistent with forearc affinity [61]. Moreover, the analyzed Al-spinels contained $\mathrm{Al}_{2} \mathrm{O}_{3}$ ranges from 33.55 to $38.55 \mathrm{wt}$.\%, and $\mathrm{Cr} \#$ from 0.34 to 0.39 , which may be related to decompression melting in the asthenosphere (Al content decreases with increases in the degree of partial melting) according to $[17,38]$.

In addition, depletion of $\mathrm{Na}_{2} \mathrm{O}$ (av. 0.12 wt.\%), $\mathrm{K}_{2} \mathrm{O}$ (av. 0.013 wt.\%), $\mathrm{Al}_{2} \mathrm{O}_{3}$ (av. 2.1 wt.\%), $\mathrm{Cr}_{2} \mathrm{O}_{3}$ (av. 0.628 wt.\%), and $\mathrm{TiO}_{2}$ (av. 0.048 wt.\%) in the examined Cpx (Figure 4c,d), and the high forsterite $(\mathrm{Fo}=91.94-98.13$, Figure $4 \mathrm{a})$ content of the analyzed olivine relics, reflect depleted mantle that had been subjected to high partial melting in a forearc setting [38].

\subsection{Protolith and Tectonic Setting}

The presence of primary orthopyroxene, olivine, and spinel relics in the examined rocks, as well as enrichment of transition elements such as Ni (1265-2632 ppm) and Co (75-170 ppm), reflect their mantle origin. Bulk major contents of $\mathrm{SiO}_{2}$ and $\mathrm{Al}_{2} \mathrm{O}_{3}$ are 
immobile during alteration processes relative to $\mathrm{CaO}$ and LFSEs; therefore, they can be used as indices of mantle depletion [44,61]. The tectonic setting of the ultramafic rocks is still a subject of debate. Some of the earliest authors suggested a mid-ocean ridge origin for the Egyptian ophiolites, based on the geochemistry of their basaltic units [63,64]. Geochemical studies of the Egyptian ophiolites recognize a supra-subduction zone, which have a geochemical nature of spreading centers in back-arc basin [65] or fore-arc basin $[8,22]$.

$\mathrm{Al}_{2} \mathrm{O}_{3}$ concentration in the bulk-rock chemistry and mineral chemistry of spinel, olivine, and pyroxene can be used to determine typical tectonic settings via various discrimination diagrams [66].

Harzburgite-dunite complex protoliths are indicated by bastite and mesh textures, in addition to high contents of compatible elements such as $\mathrm{Ni}$ and Co. Ultramafics of supra-subduction zone affinity (forearc) are characterized by low $\mathrm{TiO}_{2}$ contents $(<0.05$ wt. $\%$ ), $\mathrm{MgO} / \mathrm{SiO}_{2}$ ratios $(<1.4), \mathrm{Al}_{2} \mathrm{O}_{3}$ contents $\left(<0.78\right.$ wt. $\%$ ), and $\mathrm{Al}_{2} \mathrm{O}_{3} / \mathrm{SiO}_{2}$ ratios $(<0.02)$, according to $[17,49]$. The $\mathrm{Al}_{2} \mathrm{O}_{3} / \mathrm{SiO}_{2}$ ratios of the examined rocks show very narrow variance, ranging from 0.004 to 0.04 , with an average of 0.014 -lower than that of PM composition ( 0.1) [67], but close to that of depleted mantle harzburgite $(\sim 0.02)$ [63] suggesting that they underwent partial melting before serpentinization processes [17]. Conversely, the average of $\mathrm{MgO} / \mathrm{SiO}_{2}$ ratio of these rocks ranged from $0.81-1.14$, with an average of 0.99 -higher than that of $\mathrm{PM}(\sim 0.85) . \mathrm{Al}_{2} \mathrm{O}_{3} / \mathrm{SiO}_{2}$ and $\mathrm{MgO} / \mathrm{SiO}_{2}$ ratios were not affected during hydration processes or peridotite metamorphism [46]. The studied samples lie above the trend of mantle terrestrial arrays and abyssal peridotites straddling the forearc field (Figure 10a). In addition, they have $\mathrm{Al}_{2} \mathrm{O}_{3} / \mathrm{SiO}_{2}(0.004-0.04)$ and $\mathrm{MgO} / \mathrm{SiO}_{2}$ (av. 0.99) ratios similar to those of forearc peridotites [61]. Among the HFSEs, the studied peridotites are variably depleted in $\mathrm{Nb}$, consistent with an SSZ geochemical signature [52]. According to the $\mathrm{Zr}$ vs. $\mathrm{Nb}$ binary diagram in [68], the examined rocks plot among depleted mantle sources (Figure 10b).

On the other hand, the mineral chemistry of $\mathrm{Cr}$-spinel can be suggestive of a forearc setting. The ternary Cr-Al-Fe diagram [18] (Figure 5d), Mg\# vs. Cr\# [1] (Figure 10c), and $\mathrm{Cr} \#$ vs. $\mathrm{TiO}_{2}$ [69] (Figure 10d) reveal a forearc signature. In addition, there is a positive relationship between the $\mathrm{Cr} \#$ content of spinels and the partial degree of melting or melt/rock reaction, where those with Cr\# $>6$ are formed in supra-subduction (mostly forearc) environments with a high degree of partial melting, whilst those with Cr\# $<6$, with tholeiitic magma, are formed in abyssal zones such as mid-ocean ridges (MORs) [66,70]. The average Cr\# of the examined Cr-spinels was more than 0.6 (av. 0.689), which is consistent with those of modern forearc serpentinites [22]. Furthermore, high Fo content (av. $95.23 \mathrm{~mol} \%$ ) also reflects their forearc origins [22] (Figure 4a). The clinopyroxene compositions of the examined ultramafic rocks plot in the field characteristic of forearc settings, due to depletion of $\mathrm{Al}_{2} \mathrm{O}_{3}, \mathrm{Na}_{2} \mathrm{O}$, and $\mathrm{Cr}_{2} \mathrm{O}_{3}$ [70] (Figure 4c,d). Likewise, orthopyroxenes are moderate in $\mathrm{Al}_{2} \mathrm{O}_{3}(<3.23 \mathrm{wt} . \%)$ and low in $\mathrm{Na}_{2} \mathrm{O}\left(<0.04\right.$ wt.\%), $\mathrm{TiO}_{2}\left(<0.04\right.$ wt.\%), and $\mathrm{Cr}_{2} \mathrm{O}_{3}(<0.92 \mathrm{wt} . \%)$, consistent with forearc peridotite orthopyroxenes $[24,28]$ (Figure $4 \mathrm{e}$ ).

The current results of the examined ultramafics suggest that they are relics of the oceanic lithosphere that were emplaced in a forearc environment (Figure 11). 

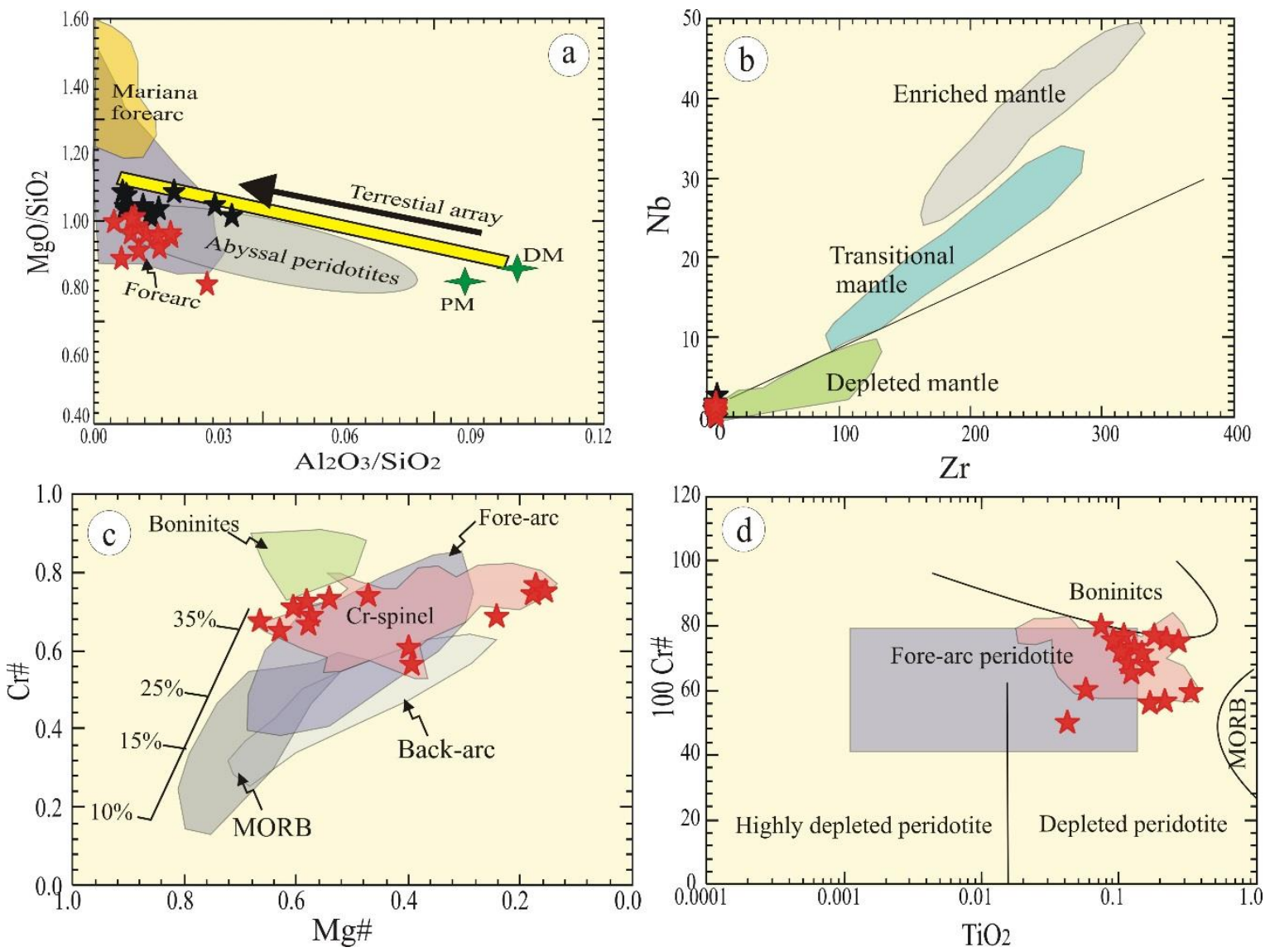

Figure 10. (a) $\mathrm{Al}_{2} \mathrm{O}_{3} / \mathrm{SiO}_{2}$ vs. $\mathrm{MgO} / \mathrm{SiO}_{2}$ in the studied ultramafic samples. Abyssal peridotite, primitive mantle, and depleted mantle are from [44,46,67], respectively. Mariana forearc and forearc are from [44,61], respectively. The thick yellow line represents a terrestrial array [71]. (b) Zr vs. Nb diagram from [68]; (c) Mg\# versus Cr\# binary diagram from [1]; partial melting degree is from [72]. (d) $100 \mathrm{Cr} \#$ vs. $\mathrm{TiO}_{2}$ of the examined spinels (Cr- and Al-spinel). Boninites, MORB, forearc peridotites, and depleted and highly depleted peridotites are obtained from [28,73-75], respectively. The pink field represents fresh Cr-spinels of the Egyptian Eastern Desert [22].

\subsection{Geothermometry}

Different thermometer methods can be used to deduce the temperature of equilibrium of the examined serpentinized ultramafics (Supplementary Materials, Table S10). These methods include olivine-spinel thermometry using the calibrations described in [76,77], as well as $\mathrm{Al}$ in orthopyroxene-olivine-spinel thermometry [75]. The obtained average temperature of the studied serpentinized peridotites using the olivine-spinel thermometry [77] method was $781{ }^{\circ} \mathrm{C}$, which is lower than the average temperature $\left(852{ }^{\circ} \mathrm{C}\right)$ calculated using the calibration described in [76]. On the other hand, the average temperatures of the studied serpentinized peridotites calculated using the calibrations described in [75] (Al content in the orthopyroxene) was $859^{\circ} \mathrm{C}$, similar to the estimated average in [76]. In comparison with Pan-African forearc peridotites using the calibration described in [77], the estimated average temperature of the studied serpentinized peridotites $\left(781^{\circ} \mathrm{C}\right)$ is very similar to those found in $\mathrm{Um}$ Khariga $\left(778^{\circ} \mathrm{C}\right)$ [78]. In addition, the average temperature of the examined rocks obtained using $\mathrm{Al}$ in orthopyroxene thermometry $\left(859^{\circ} \mathrm{C}\right)$ is close to the average of Um Khariga peridotites $\left(984^{\circ} \mathrm{C}\right)$ using the same calibration. 


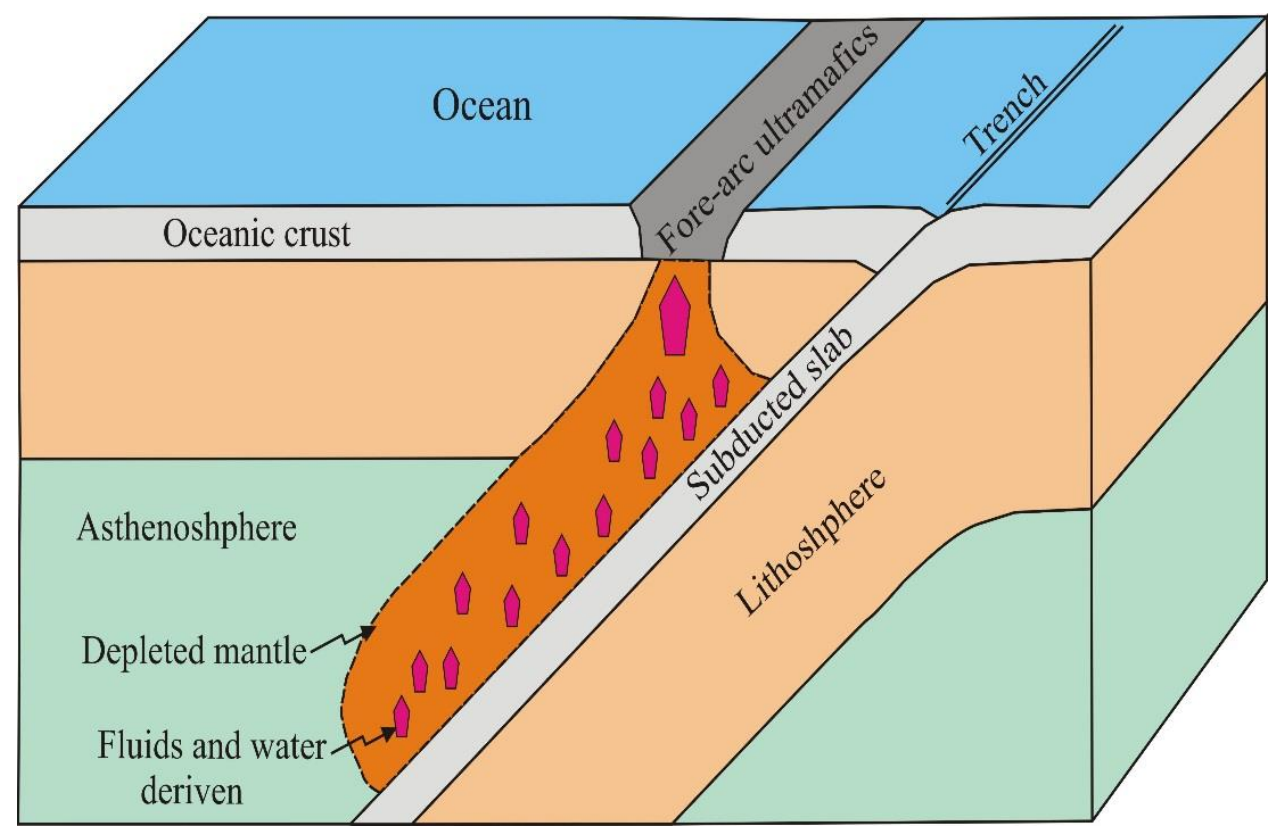

Figure 11. Schematic illustration showing the tectonic setting of the examined ultramafic rocks, modified from [79].

\section{Conclusions}

Wadi Ibib-Wadi Shani (WI-WS) ultramafics represent relics of dismembered ophiolitic rocks in the southwestern extension of the largest Gerf ophiolitic nappe, South Eastern Desert, Egypt. Based on their petrographic description, the ultramafic rocks can be divided into serpentinites and serpentinized peridotites; the former consist mainly of serpentine minerals, whereas the latter comprise serpentine minerals with relics of primary minerals such as pyroxene, $\mathrm{Cr}$-spinel, and olivine. These primary minerals are completely replaced by serpentine, magnetite, and carbonate minerals in serpentinites. The abundance of mesh and bastite textures reflects dunite and harzburgite protoliths. The composition of primary mantle minerals such as orthopyroxene $\left(\mathrm{Mg} \#\left(\mathrm{Mg} / \mathrm{Mg}+\mathrm{Fe}^{2+}\right), 89.65\right.$ to 93.18), clinopyroxene (depletion of $\mathrm{Al}_{2} \mathrm{O}_{3}, \mathrm{Na}_{2} \mathrm{O}$, and $\mathrm{Cr}_{2} \mathrm{O}_{3}$ ), high Fo content of olivine, and high Cr\# of Cr-spinel reflect a forearc setting. The calculated temperatures of the WI-WS serpentinized peridotites using olivine-spinel (av. $781^{\circ} \mathrm{C}$ ) and $\mathrm{Al}$ in orthopyroxene (av. $859^{\circ} \mathrm{C}$ ) thermometer calibrations are consistent with those of Egyptian forearc mantle rocks.

Supplementary Materials: The following are available online at https: / www.mdpi.com/article / 10.3390/app112210524/s1: Table S1: Representative microprobe analysis of orthopyroxene in serpentinized peridotites; Table S2: Representative microprobe analysis of clinopyroxene in serpentinized peridotites; Table S3: Representative microprobe analysis of Cr-spinel in serpentinized peridotites; Table S4: Representative microprobe analysis of Al-spinel in serpentinized peridotites; Table S5: Representative microprobe analysis of serpentine; Table S6: Representative microprobe analysis of chlorite in serpentinized peridotites; Table S7: Representative microprobe analysis of tremolite in serpentinized peridotites; Table S8: Representative microprobe analysis of magnetite; Table S9: Representative microprobe analysis of talc in serpentinized peridotites; Table S10: Average temperatures of serpentinized peridotites calculated using different calibrations.

Author Contributions: Conceptualization, E.S.R.L., G.M.S. and F.M.K.; methodology, E.S.R.L. and M.A.; software, E.S.R.L., G.M.S. and F.M.K.; validation, E.S.R.L., G.M.S. and F.M.K.; formal analysis, G.M.S., F.M.K. and M.A.; investigation, E.S.R.L., G.M.S. and F.M.K.; data curation, E.S.R.L., G.M.S. and F.M.K.; writing—original draft preparation, E.S.R.L., G.M.S., M.A. and F.M.K.; writing—review and editing, G.M.S., F.M.K. and E.S.R.L.; funding acquisition, M.A. All authors have read and agreed to the published version of the manuscript.

Funding: Taif University: Researchers Supporting Project grant number (TURSP-2020/196). 
Institutional Review Board Statement: Not applicable.

Informed Consent Statement: Not applicable.

Data Availability Statement: The data presented in this study are available on request from the corresponding author.

Acknowledgments: The author would like to acknowledge the financial support provided by Taif University Researchers Supporting Project Number (TURSP-2020/196).

Conflicts of Interest: The authors declare no conflict of interest.

\section{References}

1. Stern, R.J.; Johanson, P.R.; Kröner, A.; Yibas, B. Neoproterozoic ophiolites of the Arabian-Nubian Shield, Precambrian ophiolites and related rocks. In Developments in Precambrian Geology; Kusky, T.M., Ed.; Elsevier: Amsterdam, The Netherlands, 2004; pp. 95-128.

2. Dixon, T.H. The Evolution of Continental Crust in the Late Precambrian Egyptian Shield. Ph.D. Thesis, University California, San Diego, CA, USA, 1979.

3. Hamdy, M.M.; Harraz, H.Z.; Aly, G.A. Pan-African (intraplate and subduction-related?) metasomatism in the Fawakhir ophiolitic serpentinites, Central Eastern Desert of Egypt: Mineralogical and geochemical evidences. Arab. J. Geosci. 2013, 6, 13-33. [CrossRef]

4. El Bahariya, G.A. The Ophiolite-Dominated Suprastructure, Eastern Desert, Egypt. In The Geology of the Egyptian Nubian Shield. Regional Geology Reviews; Hamimi, Z., Arai, S., Fowler, A.R., El-Bialy, M.Z., Eds.; Springer: Cham, Switzerland, 2021. [CrossRef]

5. Stern, R.J. Neoproterozoic formation and evolution of Eastern Desert continental crust—the importance of the infrastructuresuperstructure transition. J. Afr. Earth Sci. 2018, 148, 15-27. [CrossRef]

6. Zoheir, B.; Abd El-Rahman, Y.; Kusky, T.; Xiong, F. New SIMS zircon U-Pb ages and oxygen isotope data for ophiolite nappes in the Eastern Desert of Egypt: Implications for Gondwana assembly. Gondwana Res. 2021. [CrossRef]

7. Abdel Halim, A.; Helmy, H.M.; Abdel-Rahman, Y.M.; Shibata, T.; El-Mahallawi, M.M.; Yoshikawa, M.; Arai, S. Petrology of the Motaghairat mafic ultramafic complex, Eastern Desert, Egypt: A high-Mg post-collisional extension-related layered intrusion. J. Asi. Earth Sci. 2016, 116, 164-180. [CrossRef]

8. El Mezayen, A.M.; Heikal, M.A.; El-Feky, M.G.; Shahin, H.A.; Abu Zeid, I.K.; Lasheen, E.S.R. Ultramafic hosting talc, chromite and uranium-bearing magnesite of Sol Hamed ophiolites South Eastern Desert, Egypt; petrology, geochemistry and tectonic evolution. Annals Geol. Surv. Egypt 2018, 35, 71-96.

9. Be'eri-Shlevin, Y.; Katzir, Y.; Whitehouse, M. Post-collisional tectonomagmatic evolution in the northern Arabian-Nubian Shield: Time constraints from ion-probe U-Pb dating of zircon. J. Geol. Soci. Lond. 2009, 166, 71-85. [CrossRef]

10. Mao, J.W.; Pirajno, F.; Zhang, Z.H.; Chai, F.M.; Wu, H.; Chen, S.P.; Cheng, L.S.; Yang, J.M.; Zhang, C.Q. A review of the Cu-Ni sulfide deposits in the Chinese Tianshan and Altay orogens (Xinjiang Autonomous Region, NW China): Principal characteristics and ore forming processes. J. Asian Earth Sci. 2008, 32, 184-203. [CrossRef]

11. Seo, J.; Oh, C.W.; Choi, S.G.; Rajesh, V.J. Two ultramafic rock types in the Hongseong area, South Korea: Tectonic significance for northeast Asia. Lithos 2013, 175-176, 30-39. [CrossRef]

12. Chen, C.; Xun Su, B.; Xiao, Y.; Uysal, I.; Lin, W.; Chu, Y.; Jing, J.J.; Sakyi, P.A. Highly siderophile elements and Os isotope constraints on the genesis of peridotites from the Kızıldağ ophiolite, southern Turkey. Lithos 2020, 368-369, 105583. [CrossRef]

13. Boskabadi, A.; Pitcairn, I.K.; Leybourne, M.I.; Teagle, D.A.H.; Cooper, M.J.; Hadizadeh, H.; Bezenjani, R.N.; Bagherzadeh, R.M. Carbonation of ophiolitic ultramafic rocks: Listvenite formation in the Late Cretaceous ophiolites of eastern Iran. Lithos 2020, 352-353, 105307. [CrossRef]

14. Kerrich, R.W. Geochemical evidence on the source of fluids and solutes for shear zone hosted mesothermal Au deposits. In Mineralization in Shear Zones; Bursnal, J.T., Ed.; Geological Association of Canada, 6 (Short Course Notes); Geological Association of Canada: St. John's, NL, Canada, 1989; pp. 129-197.

15. Shackleton, R.M. Review of late Proterozoic sutures, ophiolitic mélanges and tectonics of eastern Egypt and north Sudan. Geol. Rundschau. 1994, 83, 537-546. [CrossRef]

16. Saka, S.; Uysal, I.; Kapsiotis, A.; Bağcl, U.; Ersoy, E.Y.; Su, B.X.; Seitz, H.M.; Hegner, E. Petrological characteristics and geochemical compositions of the Neotethyan Mersin ophiolite (southern Turkey): Processes of melt depletion, refertilization, chromitite formation and oceanic crust generation. J. Asian Earth Sci. 2019, 176, 281-299. [CrossRef]

17. Deschamps, F.; Godard, M.; Guillot, S.; Hattori, K. Geochemistry of subduction zone serpentinites: A review. Lithos 2013, 178, 96-127. [CrossRef]

18. Gamal El Dien, H.M.; Hamdy, M.M.; Abu El Ela, A.; Abu Alam, T.; Hassan, A.; Kil, Y.; Mizukami, T.; Soda, Y. Neoproterozoic serpentinites from the Eastern Desert of Egypt: Insights into Neoproterozoic mantle geodynamics and processes beneath the Arabian-Nubian Shield. Precambrian Res. 2016, 286, 213-233. [CrossRef]

19. Saleh, G.M. Geochemical characteristic of some mineralization in wadi Ibib—Um Rasayn—G. Madara area, South Eastern Desert, Egypt. 2005; Report PP 57.

20. Shapiro, L.; Brannock, W.W. Rapid analysis of silicate, carbonate and phosphate rocks, U.S. Geol. Surv. Bull. 1962, 114 , A56. 
21. McDonough, W.F.; Rudnick, R.L. Mineralogy and composition of the upper mantle. In Ultrahigh Earth Sciences-Pressure mineralogy; Reviews in mineralogy 37; Hemley, R.J., Ed.; Harvard University: Cambridge, MA, USA, 1998; pp. $138-164$.

22. Azer, M.K.; Stern, R.J. Neoproterozoic (835-720 Ma) serpentinites in the Eastern Desert, Egypt: Fragments of fore-arc mantle. J. Geol. 2007, 115, 457-472. [CrossRef]

23. Morimoto, N.; Fabries, J.; Ferguson, A.K.; Ginzburg, I.V.; Ross, M.; Seifert, F.A.; Zussman, J. Nomenclature of pyroxenes. Mineral. Mag. 1988, 52, 535-550. [CrossRef]

24. Pagé, P.; Bédard, J.H.; Schroetter, J.M.; Tremblay, A. Mantle petrology and mineralogy of the Thetford Mines ophiolite complex. Lithos 2008, 100, 255-292. [CrossRef]

25. Le Bas, M.J. The role of aluminum in igneous clinopyroxenes with relation to their parentage. Amer. J. Sci. 1962, 260, 267-288. [CrossRef]

26. Nozaka, T. A note on compositional variation of olivine and pyroxene in thermally metamorphosed ultramafic complexes from SW Japan. Okayama University. Earth Sci. Rep. 2010, 17, 1-5.

27. Hattori, K.H.; Guillot, S. Geochemical character of serpentinites associated with high- to ultrahigh-pressure metamorphic rocks in the Alps, Cuba, and the Himalayas: Recycling of elements in subduction zones. Geochem. Geophys. Geosyst. 2007, 8, 9. [CrossRef]

28. Ohara, Y.; Stern, R.J.; Ishii, T.; Yurimoto, H.; Yamazaki, T. Peridotites from the Mariana Trough: First look at the mantle beneath an active back-arc basin. Contrib. Mineral. Petrol. 2002, 143, 1-18. [CrossRef]

29. Barnes, S.J.; Roeder, P.L. The range of spinel composition in terrestrial mafic ultramafic rocks. J. Petrol. 2001, 42, $2279-2302$. [CrossRef]

30. Gargiulo, M.F.; Bjerg, E.A.; Mogessie, A. Spinel group minerals in metamorphosed ultramafic rocks from Río de Las Tunas belt, Central Andes, Argentina. Geol. Acta 2013, 11, 133-148.

31. Kapsiotis, A.; Grammatikopoulos, T.A.; Tsikouras, B.; Hatzipanagiotou, K. Chromian spinel composition and platinum-group element mineralogy of chromitites from the Milia area, Pindos ophiolite complex, Greece. Can. Mineral 2009, 47, 1037-1056. [CrossRef]

32. Rajesh, H.M.; Chisonga, B.C.; Shindo, K.; Beukes, N.J.; Armstrong, R.A. Petrographic, geochemical and SHRIMP U-Pb titanite age characterization of the Thabazimbi mafic sills: Extended time frame and a unifying Petrogenetic model for the Bushveld Large Igneous Province. Precambrian Res. 2013, 230, 79-102. [CrossRef]

33. Moody, J.B. Serpentinization. A review. Lithos 1976, 9, 125-138. [CrossRef]

34. Deer, W.A.; Howie, R.A.; Zussman, J. An Introduction to the Rock Forming Minerals, 2nd ed.; Longman: London, UK, 1992; 696p.

35. Hey, M.H. A new review of the chlorite. Mineral. Mag. 1954, 30, 227. [CrossRef]

36. Cathelineau, M.; Nieva, D. A chlorite solid solution geothermometer: The Los Azufres (Mexico) geothermal system. Contrib. Mineral. Petrol. 1995, 91, 235-244. [CrossRef]

37. Zang, W.; Fyfe, W.S. Chloritization of the hydrothermally altered bedrock at the Igarape Bahia gold deposite, Carajas, Brazil. Mineral. Depos. 1995, 30, 30-38. [CrossRef]

38. Khedr, M.Z.; Arai, S. Chemical variations of mineral inclusions in Neoproterozoic high-Cr chromitites from Egypt: Evidence of fluids during chromitite genesis. Lithos 2016, 240-243, 309-326. [CrossRef]

39. Kodolanyi, J.; Pettke, T.; Spandler, C.; Kamber, B.S.; Gmeling, K. Geochemistry of Ocean Floor and Fore-arc Serpentinites: Constraints on the Ultramafic Input to Subduction Zones. J. Pertol. 2012, 53, 235-270. [CrossRef]

40. Leake, B.E. Nomenclature of amphiboles: Report of the subcommittee on amphiboles of the International Mineralogical Association, Commission on New Minerals and Mineral Names: American Mineralogist. J. Earth Planet. Mater. 1997, 82, 1019-1037.

41. Zakrutkin, V.V.; Grigorenko, M.W. Titanium and alkalies in amphiboles in metamorphism: Doklady of the Academy of Sciences U.S.S.R. Earth Sci. 1967, 173, 917-918.

42. Stromer, J.C. The effects of recalculation on estimates of temperatures and oxygen fugacity from analyses of multicomponent iron-titanium oxides. Amer. Mineral. 1983, 68, 586-594.

43. Wang, X.; Zeng, Z.; Chen, J. Serpentinization of peridotites from the southern Mariana forearc. Prog. Nat. Sci. 2009, 19, 1287-1295 [CrossRef]

44. Niu, Y. Bulk-rock major and trace element compositions of abyssal peridotites: Implications for mantle melting, melt extraction and post-melting processes beneath mid-ocean ridges. J. Petrol. 2004, 45, 2423-2458. [CrossRef]

45. Bodinier, J.L.; Godard, M. Orogenic, ophiolitic, and abyssal peridotites. In The Mantle and Core: Treatise on Geochemistry; Carlson, R.W., Ed.; Elsevier: Amsterdam, The Netherlands, 2003; pp. 103-170.

46. Salters, V.J.M.; Stracke, A. Composition of the depleted mantle. Geochem. Geophy. Geosyst. 2004, 5, 1-27. [CrossRef]

47. Palme, H.; O'Neill, H. Cosmochemical estimates of Mantle Composition. In Treatise on Geochemistry; Holland, H.D., Turrekian, K.K., Eds.; Elsevier: Amsterdam, The Netherlands, 2004; Volume 2, pp. 1-38.

48. Floyd, P.A. Oceanic Basalts; Blachie and Son Ltd.: New York, NY, USA, 1991.

49. Ishii, T.; Robinson, P.T.; Maekawa, H.; Fiske, R. Petrological studies of peridotites from diapiric serpentinite seamounts in the Izu-Ogasawara-Mariana fore-arc, LEG125. In Proceedings of the Ocean Drilling Program: Scientific Results: Ocean Drilling Program; Fryer, P., Pearce, J.A., Stokking, L.B., Eds.; Ocean Drilling Program: College Station, TX, USA, 1992; Volume 125, pp. 445-485.

50. Li, X.P.; Rahn, M.; Bucher, K. Serpentinites of the Zermatt-Saas ophiolite complex and their texture evolution. J. Metamorph. Geol. 2004, 22, 159-177. [CrossRef] 
51. Sun, S.S.; McDonough, W.F. Chemical and Isotopic Systematics of Oceanic Basalts: Implications for Mantle Composition and Processes. Geol. Soc. Lond. Spec. Publ. 1989, 42, 313-345. [CrossRef]

52. Hawkins, J.W. Geology of supra-subduction zones-implications for the origin of ophiolites. Ophiolite Concept Evol. Geol. Thought 2003, 373, 227-268.

53. Rucklidge, J.C.; Patterson, G.C. The role of Chlorine in serpentinization. Contr. Min. Petrol. 1977, 65, 39-44. [CrossRef]

54. Hamdy, M.; Gamal El Dien, H. Nature of serpentinization and carbonation of ophiolitic peridotites (Eastern Desert, Egypt): Constrains from stable isotopes and whole-rock geochemistry. Arab. J. Geosci. 2017, 10, 429. [CrossRef]

55. Evans, B.W.; Johannes, W.; Oterdsom, H.; Trommsdorff, V. Stability of chrysotile and antigorite in serpentinite multisystem. Schweiz. Mineral. Und Petrogr. Mitt. 1976, 56, 79-93.

56. Evans, B.W.; Hattori, K.; Baronnet, A. Serpentinite: What, why, where? Elements 2013, 9, 99-106. [CrossRef]

57. Polat, A.; Hofmann, A.W. Alteration and geochemical patterns in the 3.7-3.8 Ga Isua greenstone belt, West Greenland. Precambrian Res. 2003, 126, 197-218. [CrossRef]

58. Uner, T. Listwaenitization and enrichment of precious metals in the hydrothermal mineralization zones of serpentinites in Sugeçer-Van (Eastern Anatolia, Turkey). Geochem. Explor. Environ. Anal. 2019, 25. [CrossRef]

59. Uysal, I.; Ersoy, E.Y.; Karslı, O.; Dilek, Y.; Sadıklar, M.Y.; Ottley, C.J.; Tiepolo, M.; Meisel, T. Coexistence of abyssal and ultra-depleted SSZ type mantle peridotites in a Neo-Tethyan Ophiolite in SW Turkey: Constraints from mineral composition, whole-rock geochemistry (major-trace-REE-PGE) and Re-Os isotope systematic. Lithos 2012, 60, 132-133. [CrossRef]

60. Hellebrand, E.; Snow, J.E.; Dick, H.J.; Hofmann, A.W. Coupled major and trace elements as indicators of the extent of melting in mid-ocean-ridge peridotites. Nature 2001, 410, 677-681. [CrossRef]

61. Pearce, J.A.; Barker, P.F.; Edwards, S.J.; Parkinson, I.J.; Leat, P.T. Geochemistry and tectonic significance of peridotites from the South Sandwich arc-basin system, south Atlantic. Contrib. Miner. Petrol. 2000, 139, 36-53. [CrossRef]

62. Xu, X.Z.; Yang, J.S.; Ba, D.Z. Petrogenesis of the Kanginla Peridotite in the Luobusa Ophiolite, Southern Tibet. J. Asian Earth Sci. 2011, 42, 553-568. [CrossRef]

63. Tsoungui, P.N.E.; Ganno, S.; Njiosseu, E.L.T.; Mbongue, J.L.N.; Woguia, B.K.; Tamehe, L.S.; Wambo, J.D.T.; Nzenti, J.P. Geochemical constraints on the origin and tectonic setting of the serpentinized peridotites from the Paleoproterozoic Nyong series, Eseka area, SW Cameroon. Acta Geochim. 2019, 39, 402-422. [CrossRef]

64. Zimmer, M.; Kröner, A.; Jochum, K.P.; Reischmann, T.; Todt, W. The Gabal Gerf complex: A Precambrian N-MORB ophiolite in the Nubian Shield, NE Africa. Chem. Geol. 1995, 123, 29-51. [CrossRef]

65. Farahat, E.S.; El-Mahalawi, M.M.; Hoinkes, G. Continental back-arc basin origin of some ophiolites from the Eastern Desert of Egypt. Mineral. Petrol. 2004, 82, 81-104. [CrossRef]

66. Robinson, P.T.; Trumbull, R.B.; Schmitt, A.; Yang, J.S.; Li, J.W.; Zhou, M.F.; Xiong, F. The origin and significance of crustal minerals in ophiolitic chromitites and peridotites. Gondwana Res. 2015, 27, 486-506. [CrossRef]

67. McDonough, W.F.; Sun, S.S. The composition of the Earth. Chem. Geo. 1995, 120, 223-253. [CrossRef]

68. Geng, H.Y.; Sun, M.; Yuan, C.; Zhao, G.C.; Xiao, W.J. Geochemical and geochronological study of early carboniferous volcanic rocks from the West Junggar: Petrogenesis and tectonic implications. J. Asian Earth Sci. 2011, 42, 854-866. [CrossRef]

69. Arai, S. Chemistry of chromian spinel in volcanic rocks as a potential guide to magma chemistry. Mineral. Mag. 1992, 56, 173-184. [CrossRef]

70. Beccaluva, L.; Coltori, M.; Giunta, G.; Siena, F.; Tethyan, S. Cordilleran ophiolites: A reappraisal of distinctive tectono-magmatic features of supra-subduction complexes in relation to subduction mode. Tectonophysics 2004, 393, 163-174. [CrossRef]

71. Jagoutz, E.; Palme, H.; Baddenhausen, H.; Blum, K.; Cendales, M.; Dreibus, G.; Spettel, B.; Lorenz, V.; Vanke, H. The abundance of major, minor and trace elements in the earth's mantle as derived from primitive ultramafic nodules. Geochim. Cosmochim. Acta 1979, 11, 2031-2050.

72. Hirose, K.; Kawamoto, T. Hydrous partial melting of lherzolite at $1 \mathrm{GPa}$ : The effect of $\mathrm{H} 2 \mathrm{O}$ on the genesis of basaltic magmas. Earth Planet. Sci. Lett. 1995, 133, 463-473. [CrossRef]

73. Dick, H.J.; Bullen, T. Chromian spinel as a petrogenetic indicator in abyssal and alpine type peridotites and spatially associated lavas: Contrib. Mineral. Petrol. 1984, 86, 54-76. [CrossRef]

74. Jan, M.Q.; Windley, B.F. Chromian spinel-silicate chemistry in ultramafic rocks of the Jijal complex, Northwestern Pakistan. J. Petrol. 1990, 31, 667-715. [CrossRef]

75. Witt-Eickschen, G.E.; Seck, H.A. Solubility of $\mathrm{Ca}$ and $\mathrm{Al}$ in orthopyroxene from spinel peridotite: An improved version of an empirical geothermometer. Contrib. Miner. Petrol. 1991, 106, 431-439. [CrossRef]

76. O'Neill, H.; Wall, V.J. The Olivine-Orthopyroxene-Spinel Oxygen Geobarometer, the Nickel Precipitation Curve, and the Oxygen Fugacity of the Earth's Upper Mantle. J. Petrol. 1987, 28, 1169-1191. [CrossRef]

77. Ballhaus, C.; Berry, R.F.; Green, D.H. High pressure experimental calibration of the olivine-orthopyroxene-spinel oxygen geobarometer: Implications for the oxidation state of the upper mantle. Contrib. Mineral. Petrol. 1991, 107, 27-40. [CrossRef]

78. Ali, R.M.; Pitcairn, I.K.; Maurice, A.E.; Azer, M.K.; Bakhit, B.R.; Shahien, M.G. Petrology and geochemistry of ophiolitic ultramafic rocks and chromitites across the Eastern Desert of Egypt: Insights into the composition and nature of a Neoproterozoic mantle and implication for the evolution of SSZ system. Precambrian Res. 2020, 337, 105-565. [CrossRef]

79. Furnes, H.; Safonova, I. Ophiolites of the Central Asian Orogenic Belt: Geochemical and petrological characterization and tectonic settings. Geosci. Front. 2019, 10, 1255-1284. [CrossRef] 